\title{
Global Optimization for Scheduling Refinery Crude Oil Operations
}

\author{
Ramkumar Karuppiah $^{\mathrm{a}}$, Kevin C. Furman ${ }^{\mathrm{b}}$ and Ignacio E. Grossmann ${ }^{\mathrm{a}^{*}}$ \\ a Department of Chemical Engineering, Carnegie Mellon University, Pittsburgh, PA \\ 15213, U.S.A. \\ ${ }^{\mathrm{b}}$ ExxonMobil Research and Engineering, Annandale, NJ 08801, U.S.A.
}

April 2007

\begin{abstract}
In this work we present an Outer-Approximation algorithm to obtain the global optimum of a nonconvex Mixed Integer Nonlinear Programming (MINLP) model for the scheduling of crude oil movement at the front-end of a petroleum refinery. The model relies on a continuous time representation making use of transfer events. The proposed technique focuses on effectively solving a Mixed Integer Linear Programming (MILP) relaxation of the nonconvex MINLP to obtain a rigorous lower bound on the global optimum. Cutting planes derived by spatially decomposing the network are added to the MILP relaxation of the original nonconvex MINLP in order to tighten the lower bound and reduce the solution times for the MILP relaxation. The solution of this problem is used as a heuristic to obtain a feasible solution to the MINLP which serves as an upper bound. The lower and upper bounds are made to converge to within a specified tolerance in the proposed Outer Approximation algorithm. On applying the proposed technique on test examples, significant savings were realized in the computational effort required to obtain the globally optimal solutions and to verify their global optimality.
\end{abstract}

\footnotetext{
*Corresponding author. Tel.: +1-412-268-2230; Fax: +1-412-268-7139. Email address: grossmann@cmu.edu (I.E. Grossmann)
} 
Keywords: Refinery scheduling; Nonconvex MINLP; Global optimization; Spatial decomposition

\section{Introduction}

Scheduling and planning of the flow of crude oil is a very important problem in a petroleum refinery due to the potential realization of large cost savings and improved feeds. Linear programming (LP) models have been historically used in the analysis of scheduling and planning problems due to their ease of modeling and solution. Refinery planning problems have been addressed using computational tools such as AspenTech ${ }^{\circledR}$ PIMS (Process Industry Modeling System) that are largely based on Successive Linear Programming. However, it is difficult to model refinery operations since they involve units operating in both batch and continuous modes along with multiple grades of crude oil and products. Furthermore, detailed scheduling models often require a continuous time representation and a more general treatment of nonlinear equations, as well as binary variables to model discrete decisions which give rise to Mixed Integer Nonlinear Programming (MINLP) models. These models impart additional flexibility to the problem allowing the modeling of discrete decisions and constraints.

There are two major approaches for modeling scheduling problems: discrete time formulations and continuous time formulations (Mendez et al., 2006). In discrete time models, it is relatively easy to model the material balances and the flow constraints. However, the number of time intervals required for an accurate representation of the system is usually very high, thus the resulting models are large in size and computationally challenging. Continuous time models are smaller in comparison and allow for a complete utilization of the time domain, although it is difficult to synchronize the material balances and time sequencing constraints in such a representation. Lee et al. (1996) have proposed a Mixed Integer Linear Programming (MILP) model for short term scheduling of crude oil using discrete time intervals. Here, they derive a linear approximation of the nonlinear mixing operations by replacing bilinear terms in the mass balances by individual component flows. An MILP model has also been developed by Shah (1996) for crude oil scheduling where the scheduling time horizon is discretized into intervals of equal duration, where the requirement is that the operations must start 
and end at the boundaries of the intervals. This approach is more restricted as compared to that of Lee et al. (1996) since the front end of the refinery is decomposed into two parts - downstream and upstream, and the models corresponding to these are solved sequentially. A continuous time formulation has been used by Jia et al. (2003) where the authors present an MILP model developed by relaxing the nonlinear mixing constraints. They also include the possiblity of incorporating the bilinear equations, thus making the model an MINLP formulation. A rigorous extension of this model can be found in Furman et al. (2006), where the authors use a continuous time event formulation to schedule fluid transfer between tanks, and model the problem as an MINLP. In this work, the main idea is to allow both inputs and outputs for a tank in a single transfer event. A comparison of the discrete and continuous time formulations for scheduling for chemical processes can be found in Floudas and Lin (2004).

In this work, we apply a novel continuous time formulation given by Furman et al. (2006) to model the literature test cases given in Lee et al. (1996) for short-term scheduling of crude oil at the front-end of a refinery as an MINLP. This scheduling problem involves crude oil unloading from a crude supply source to the crude storage tanks, transfer of crude from these tanks to the charging tanks, and charging the crude distillation units continuously over a time horizon, with crude mixes from the charging tanks. We assume that a crude supply plan is in place where we know the crude arrival times and the corresponding arrival quantities and compositions.

The MINLP corresponding to the scheduling problem is nonconvex due to the presence of bilinear terms in some of the mass balance constraints, and hence the standard methods for solving MINLPs (see Grossmann, 2002) may fail to converge to a solution or lead to sub-optimal solutions. Branch and bound based methods have been reported in the literature (Sahinidis, 1996; Adjiman et al., 2000) for globally optimizing nonconvex models. The Outer Approximation algorithms developed by Duran and Grossmann (1986) and by Fletcher and Leyffer (1994) can yield globally optimal solutions only if the feasible space and the objective function of the problem are both convex. For nonconvex MINLPs, a finitely convergent decomposition algorithm based on Outer Approximation has been proposed, for instance, by Kesavan et al. (2004) to solve these MINLPs to global optimality. Nonconvexities have also been handled by Bergamini 
et al. (2005), who have presented a global optimization algorithm for Generalized Disjunctive Programming (GDP) problems. A further extension of the basic idea of Outer Approximation for the global optimization of deterministic and stochastic nonconvex MINLPs can be found in Wei et al. (2005).

In this work, we present an Outer-Approximation algorithm to obtain globally optimal solutions of the nonconvex MINLPs (with binary integer variables only) arising in the scheduling of crude oil movement in a petrochemical refinery, where the objective is to minimize the costs involved in the operation and in maintaining the inventory levels in the crude tanks. The proposed technique focuses on effectively solving the MILP relaxation of the nonconvex MINLP to obtain a tight and rigorous lower bound on the solution of the MINLP. Based on a decomposition of the original MINLP model, we generate sub-models whose solutions are used to derive valid cutting planes. These cuts are added to the MILP relaxation of the original problem in order to tighten the relaxation and reduce the computational expense of solving the relaxations. Numerical examples are presented to demonstrate that the use of such an algorithm on a class of nonconvex MINLPs can result in significant computational savings.

This paper is organized as follows. Section 2 presents the problem statement of the crude scheduling problem while section 3 provides the nonconvex MINLP model. A discussion of the algorithm is given in section 4 . Section 5 presents the different examples on which the algorithm was applied, and finally, section 6 summarizes some conclusions and recommendations for future work.

\section{Problem Statement}

The front-end of a refinery is a network consisting of supply streams, storage tanks, charging tanks and crude distillation units (CDUs) whose structure is shown in Fig. 1. The supply streams are connected to the storage tanks which are connected to the charging tanks, which in turn, are connected to the CDUs. The supply streams, which are crude carrying vessels, deliver crude oil to the storage tanks (intermediate tanks), which transfer the crude to the charging tanks. Different qualities of crude get blended into 
various crude mixtures inside the charging tanks, which are then charged directly to the distillation units.

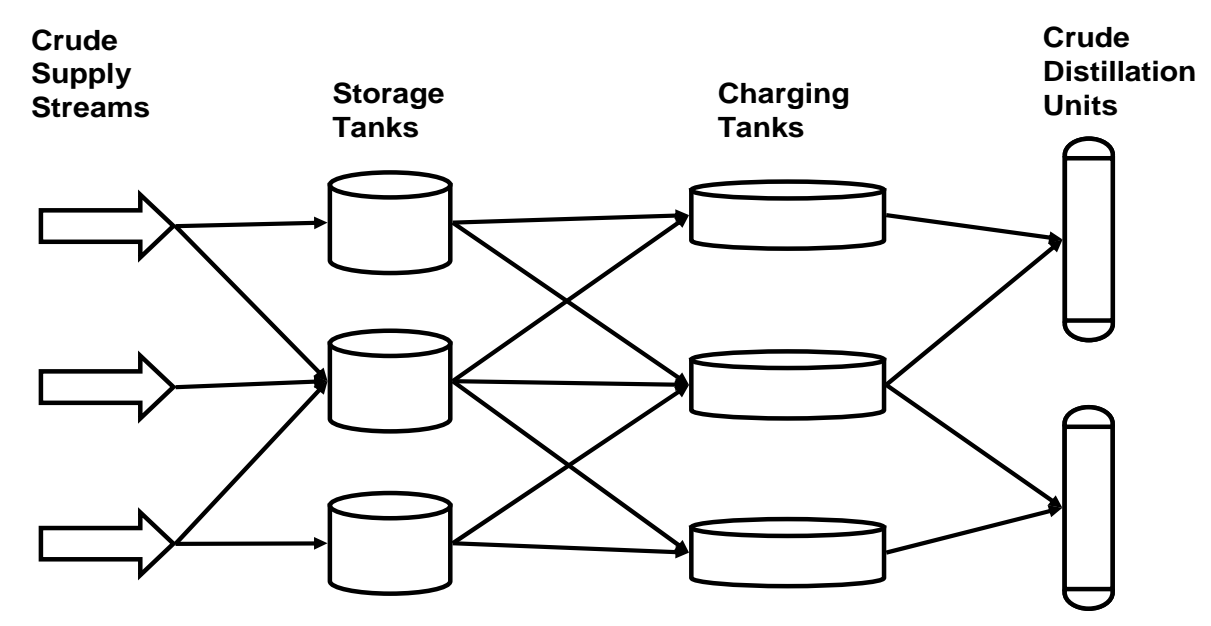

Fig. 1 Schematic of the front-end of a refinery

For scheduling the flow of crude oil in the above network, the following information is given:

(a) The maximum and minimum inventory levels for a tank (capacity limitations); (b) the initial total and component inventories in a tank; (c) upper and lower bounds on the fraction of key components in the crude inside a tank (crude quality limitations); (d) times of arrival of crude oil in the supply streams; (e) amount of crude arriving in the supply streams; (f) fractions of various components in the supply streams; (g) demand of crude-mix to be charged from a charging tank; (h) bounds on the flowrates of the streams in the network; (i) time horizon for scheduling; ( $j$ ) cost coefficients for calculating the various costs involved.

The problem is then to determine the optimum values of the following items in the system in order to minimize the total operating cost of the network: (i) the total and component inventory levels in the tanks at various instances of time; (ii) the total and component flow volumes from one unit to another in a certain time interval; (iii) start and end times of the flows in each stream present in the network.

Finally, the following operating constraints must hold in the network: 
1. Simultaneous inputs into and outputs from a tank cannot be allowed. This is done to allow settling of the crude mix in a tank.

2. Each distillation unit may be charged by at most one charging tank over a period of time. This is another operational norm followed in in many refineries.

3. Each charging tank may charge at most one distillation unit at a point of time.

4. Each charging tank has to discharge a specified amount of crude-mix to the various distillation units within the given time horizon.

5. All the distillation units have to be operated continuously throughout the entire time horizon.

\section{Model}

We model the optimization of the network as a nonconvex MINLP problem. Certain assumptions are made prior to modeling the system:

1. Perfect mixing takes place in each tank.

2. Negligible change in specific gravities on mixing.

3. The crude flows into and from a tank need not be continuous.

4. Changeover times for CDU charging are neglected.

The mathematical model for the scheduling problem has largely been taken from Furman et al. (2006) and it mainly involves mass balances, sequencing constraints, allocation constraints, and crude supply and demand constraints. This is a continuous time model for scheduling for which a number of transfer events are postulated for the transfer of material between units in the network over a given time horizon, as shown in Fig. 2. Note that as opposed to most scheduling models (see Mendez et al., 2006), the times here involve timings of transfer between pairs of units.

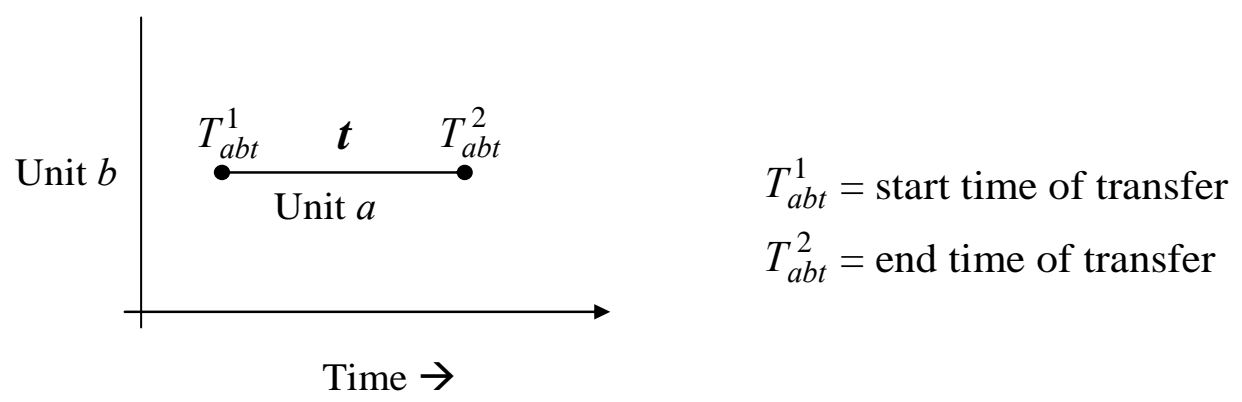

Fig. 2 Timing for transfer from unit ' $a$ ' to unit ' $b$ ' in event ' $t$ ' 
When fluid transfers take place between tanks $a$ and $b$, these are assumed to take place over the same transfer event $t$, and for which precedence constraints are imposed for the start and end times that are unknown. The number of transfer events needed to characterize the time horizon for each stream is not known as in other continuous time models, and is chosen arbitrarily before the optimization. A higher number of transfer events leads to a better representation of the schedule, although it increases the size of the model. The novelty in the model lies in the fact that inputs and outputs are allowed to occur in a single transfer event. However, simultaneous input and output is not allowed for any tank in the same transfer event and therefore all input flows must finish before an output flow starts for any tank in any transfer event. This kind of formulation reduces the number of binary variables required in the model. The optimization model consists of constraints for the crude tanks, for the distillation units, and for the supply streams:

\section{Tank Constraints}

(i) Constraints for flow transfers

$$
\begin{array}{ll}
V_{a b t}^{t o t} \leq V_{a b}^{U} w_{a b t} & \forall a \in A_{b}, \forall b \in B, \forall t \in T \\
V_{b c t}^{t o t} \leq V_{b c}^{U} w_{b c t} & \forall c \in C_{b}, \forall b \in B, \forall t \in T
\end{array}
$$

These constraints force the total flow in a stream $\left(V_{a b t}^{t o t}\right)$ from a source tank $a$ to any destination tank $b$ in a particular transfer event $t$ to zero if the binary variable, $w_{a b t}$, which pertains to the existence of flow in that stream in transfer event $t$, takes a value of zero. Note that the first subscript denotes the source from where the flow is taking place, while the second subscript denotes the destination to where the flow is going. The third and final subscript denotes the transfer event when the particular flow occurs. The binary variable $w_{a b t}$ represents the existence of flow between source $a$ and tank $b$ in transfer event $t$. The same is true for binary variable $w_{b c t}$ which takes on a value of 1 or 0 , respectively, depending on whether or not there is flow between tank $b$ and a destination unit $c$ in transfer event $t$. The first subscript in the binary variable $w$, stands for the source of the flow, while the second subscript denotes the destination of the flow. The third and final subscript stands for the transfer event in which the flow is taking place. 


\section{(ii) Duration constraints}

$$
\begin{aligned}
& F_{a b}^{U}\left(T_{a b t}^{2}-T_{a b t}^{1}\right)+F_{a b}^{U} H\left(1-w_{a b t}\right) \geq V_{a b t}^{t o t} \quad \forall a \in A_{b}, \forall b \in B, \forall t \in T \\
& F_{b c}^{U}\left(T_{b c t}^{2}-T_{b c t}^{1}\right)+F_{b c}^{U} H\left(1-w_{b c t}\right) \geq V_{b c t}^{t o t} \quad \forall c \in C_{b}, \forall b \in B, \forall t \in T
\end{aligned}
$$

For a flow between source $a$ and tank $b$, the timing variables $T_{a b t}^{1}$ and $T_{a b t}^{2}$ correspond to the start and end times of flow in a stream from $a$ to $b$ in transfer event $t$. The timing variables $T_{b c t}^{1}$ and $T_{b c t}^{2}$ are similarly defined for a flow between tank $b$ and a destination $c$ in transfer event $t$. $H$ is the overall time horizon of operation. These constraints are relaxed and the timing variables can take on any value if there is no flow in a certain transfer event. The above is expressed through big-M constraints that state that, if there is a flow in a stream in the network in transfer event $t$, the product of the upper bound on the flowrate of the crude stream with the duration of flow in the transfer event gives an upper bound on the total flow volume in that transfer event.

$$
\begin{aligned}
& F_{a s}^{L}\left(T_{a s t}^{2}-T_{a s t}^{1}\right)-F_{a s}^{L} H\left(1-w_{a s t}\right) \leq V_{a s t}^{t o t} \quad \forall a \in A_{s}, \forall s \in S, \forall t \in T \\
& F_{s c}^{L}\left(T_{s c t}^{2}-T_{s c t}^{1}\right)-F_{s c}^{L} H\left(1-w_{s c t}\right) \leq V_{s c t}^{\text {tot }} \quad \forall c \in C_{s}, \forall s \in S, \forall t \in T \\
& F_{g c}^{L}\left(T_{g c t}^{2}-T_{g c t}^{1}\right) \leq V_{g c t}^{t o t} \quad \forall c \in C_{g}, \forall g \in G, \forall t \in T
\end{aligned}
$$

Similarly, as given in eq (4a) and eq (4b), if there is a flow in transfer event $t$ into or from a tank, the lower bound on the volume of a flow is obtained by multiplying the fluid flowrate lower bound with the duration of flow. We should note that for the charging tanks, the start and end times have to coincide if there is no flow in a particular time event (eq (11b)). This enforces the continuity of operation of the CDUs under the condition that only one charging tank can charge a CDU in a certain transfer event.

(iii) Simple sequencing constraints

A flow into or from a tank $b$ in transfer event $t$ has to take place before the same flow in event $t+1$. Equations (5) - (10) correspond to this necessary condition.

$$
\begin{array}{ll}
T_{a b, t+1}^{1} \geq T_{a b t}^{2}-H\left(1-w_{a b t}\right) & \forall a \in A_{b}, \forall b \in B, \forall t \in T, t<|T| \\
T_{a b, t+1}^{1} \geq T_{a b t}^{1} & \forall a \in A_{b}, \forall b \in B, \forall t \in T, t<|T|
\end{array}
$$




$$
\begin{array}{ll}
T_{a b, t+1}^{2} \geq T_{a b t}^{2} & \forall a \in A_{b}, \forall b \in B, \forall t \in T, t<|T| \\
T_{b c, t+1}^{1} \geq T_{b c t}^{2}-H\left(1-w_{b c t}\right) & \forall c \in C_{b}, \forall b \in B, \forall t \in T, t<|T| \\
T_{b c, t+1}^{1} \geq T_{b c t}^{1} & \forall c \in C_{b}, \forall b \in B, \forall t \in T, t<|T| \\
T_{b c, t+1}^{2} \geq T_{b c t}^{2} & \forall c \in C_{b}, \forall b \in B, \forall t \in T, t<|T|
\end{array}
$$

If no flow exists between $a$ and $b$ in transfer event $t$ (i.e. $w_{a b t}=0$ ) then the bigM inequality (5) is relaxed. Similarly, if there is no flow from $b$ to $c$ in the transfer event $t$ (i.e. $w_{b c t}=0$ ) then the big-M inequality (8) is relaxed. Essentially, it means that if there is no flow in a stream in a transfer event $t$, then the values taken by the variables pertaining to the start and end times of flow in transfer event $t$ are meaningless and do not affect the flow times in the next transfer event when there is flow.

(iv) Input and output restraints for the entire horizon

A set of constraints have to enforce the condition that any inputs or outputs of the current transfer event $t$ must occur after the inputs and outputs of the preceding transfer event. The inclusion of these time constraints, which are expressed as big-M constraints, enforces the material balances to be calculated properly across all tanks in the same transfer event.

$$
\begin{array}{ll}
T_{a b, t+1}^{1} \geq T_{a^{\prime} b t}^{2}-H\left(1-w_{a^{\prime} b t}\right) & \forall a, a^{\prime} \in A_{b}, a \neq a^{\prime}, \forall b \in B, \forall t \in T, t<|T| \\
T_{a b, t+1}^{1} \geq T_{b c t}^{2}-H\left(1-w_{b c t}\right) & \forall a \in A_{b}, \forall c \in C_{b}, \forall b \in B, \forall t \in T, t<|T| \\
T_{b c, t+1}^{1} \geq T_{a b t}^{2}-H\left(1-w_{a b t}\right) & \forall a \in A_{b}, \forall c \in C_{b}, \forall b \in B, \forall t \in T, t<|T| \\
T_{b c, t+1}^{1} \geq T_{b c^{\prime} t}^{2}-H\left(1-w_{b c^{\prime} t}\right) & \forall c, c^{\prime} \in C_{b}, c \neq c^{\prime}, \forall b \in B, \forall t \in T, t<|T|
\end{array}
$$

Also since all inputs into a tank $b$ are required to finish before any output starts from that tank $b$ in any transfer event, we need the following constraint:

$$
T_{a b t}^{2}-H\left(1-w_{a b t}\right) \leq T_{b c t}^{1}+H\left(1-w_{b c t}\right) \quad \forall a \in A_{b}, \forall c \in C_{b}, \forall b \in B, \forall t \in T
$$

This helps in upholding material balances in the transfer event $t$ and prevents the situation where output could occur before any input into a tank.

(v) Mass balances

$$
\begin{aligned}
& I_{b, t-1}^{\text {tot }}+\sum_{a \in A_{b}} V_{a b t}^{\text {tot }}=I_{b t}^{\text {tot }}+\sum_{c \in C_{b}} V_{b c t}^{\text {tot }} \quad \forall t \in T, \forall b \in B \\
& I_{b 0}^{\text {tot }}=I_{b}^{\text {init-tot }} \quad \forall b \in B
\end{aligned}
$$




$$
\begin{aligned}
& I_{j b, t-1}+\sum_{a \in A_{b}} V_{j a b t}=I_{j b t}+\sum_{c \in C_{b}} V_{j b c t} \quad \forall j \in J, \forall t \in T, \forall b \in B \\
& I_{j b 0}=I_{j b}^{i n i t} \quad \forall j \in J, \forall b \in B \\
& V_{a b t}^{t o t}=\sum_{j \in J} V_{j a b t} \quad \forall a \in A_{b}, \forall t \in T, \forall b \in B \\
& V_{b c t}^{t o t}=\sum_{j \in J} V_{j b c t} \quad \forall c \in C_{b}, \forall t \in T, \forall b \in B
\end{aligned}
$$

For each tank $b \in \mathrm{B}$ in the network, we have an overall inventory balance (eq (13)), individual inventory balances (eq (15)) for each component $j \in \mathrm{J}$ and the total flow balances (eq (17) and eq (18)). The inventory balances imply that the inventory in tank $b$ at the end of a transfer event $t$ is equal to the inventory at the end of transfer event $t$ - 1 plus the volume flow into the tank from any input source $a$ in transfer event $t$, minus the flow to any output destination $c$ in the transfer event $t$. The representation of a crude tank is shown in Fig. 3.

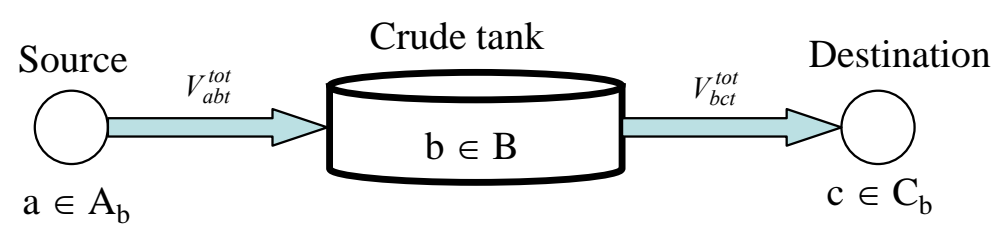

\section{Fig. 3 Crude tank representation}

The variables $I_{b t}^{\text {tot }}$ and $I_{j b t}$ correspond to the total inventory and individual component inventory in a tank $b$ at the end of transfer event $t$, respectively. The volume flow balances imply that the total flow into or out from a tank equals the sum of the individual component flows. $V_{a b t}^{\text {tot }}$ stands for the total volume flow from any source $a$ to tank $b$ in transfer event $t$, while $V_{b c t}^{\text {tot }}$ represents the flow from tank b to a destination $c$ to which this tank is connected. $V_{j a b t}$ and $V_{j b c t}$ are the respective component flows.

\section{(vi) Component balances}

On assuming perfect mixing in a tank, the fraction of a component $j$ in the output flow from a tank should be equal to the fraction of that component 
present inside the tank. This constraint is formulated as follows, with bilinear terms, which give rise to the nonconvexity of the model:

$$
\left(I_{b, t-1}^{t o t}+\sum_{a \in A_{b}} V_{a b t}^{t o t}\right) V_{j b c t}=\left(I_{j b, t-1}+\sum_{a \in A_{b}} V_{j a b t}\right) V_{b c t}^{t o t} \quad \forall j \in J, \forall t \in T, \forall c \in C_{b}, \forall b \in B
$$

(vii) Inventory bounds

The following constraint must hold in order to ensure that the total inventory in any transfer event does not exceed the upper bound of the inventory since both inputs and outputs can occur in the same transfer event.

$$
I_{b, t-1}^{t o t}+\sum_{a \in A_{b}} V_{a b t}^{t o t} \leq I_{b}^{U} \quad \forall b \in B, \forall t \in T
$$

The sum $\left(I_{b, t-1}^{t o t}+\sum_{a \in A_{b}} V_{a b t}^{t o t}\right)$ is the total inventory in a tank $b$ in transfer event $t$ before any output flow starts to occur from the tank in the same transfer event.

(viii) Bounds on components fractions inside a tank

The fraction of a component in the crude inside any tank should lie between given bounds. This is enforced by the following constraints:

$$
\begin{aligned}
& f_{j b}^{L} I_{b t}^{\text {tot }} \leq I_{j b t} \leq f_{j b}^{U} I_{b t}^{\text {tot }} \quad \forall j \in J, \forall b \in B, \forall t \in T \\
& f_{j b}^{L} V_{b c t}^{\text {tot }} \leq V_{j b c t} \leq f_{j b}^{U} V_{b c t}^{\text {tot }} \quad \forall j \in J, \forall b \in B, \forall c \in C_{b}, \forall t \in T
\end{aligned}
$$

$f_{j b}^{L}$ and $f_{j b}^{U}$ stand for the lower and upper bounds, respectively, of the fraction of a component $j$ inside a tank $b$.

(ix) Crude-mix demand constraints

Each charging tank $g \in \mathrm{G}$ must charge a specified amount of crude-mix over the entire scheduling horizon. This volume of crude-mix is distributed to the different CDUs in the network.

$$
\sum_{d \in D_{g}} \sum_{t} V_{g d t}^{t o t}=D M_{g} \quad \forall g \in G
$$

(x) Bound strengthening cuts (optional)

The following constraints may be added to the model in an attempt to tighten the relaxation of the MINLP model so as to accelerate the convergence to find the optimal solution. These are derived using a reformulation and 
linearization technique given in Sherali and Alameddine (1992). In this we take eq (19) and expand it to get the following equation:

$$
I_{b, t-1}^{t o t} V_{j b c t}+\sum_{a \in A_{b}} V_{a b t}^{t o t} V_{j b c t}=I_{j b, t-1} V_{b c t}^{t o t}+\sum_{a \in A_{b}} V_{j a b t} V_{b c t}^{t o t} \quad \forall j \in J, \forall t \in T, \forall c \in C_{b}, \forall b \in B
$$

Each bilinear term present in the above equation is considered and a summation is carried out over $j \in \mathrm{J}$ for each of these bilinear terms, which results in the following set of equations,

$$
\begin{array}{ll}
\sum_{j \in J} I_{b, t-1}^{t o t} V_{j b c t}=I_{b, t-1}^{t o t} V_{b c t}^{t o t} & \forall t \in T, \forall c \in C_{b}, \forall b \in B \\
\sum_{j \in J} V_{a b t}^{t o t} V_{j b c t}=V_{a b t}^{t o t} V_{b c t}^{t o t} & \forall a \in A_{b}, \forall t \in T, \forall c \in C_{b}, \forall b \in B \\
\sum_{j \in J} I_{j b, t-1}^{t o t} V_{b c t}^{t o t}=I_{b, t-1}^{t o t} V_{b c t}^{t o t} & \forall t \in T, \forall c \in C_{b}, \forall b \in B \\
\sum_{j \in J} V_{j a b t} V_{b c t}^{t o t}=V_{a b t}^{t o t} V_{b c t}^{t o t} & \forall a \in A_{b}, \forall t \in T, \forall c \in C_{b}, \forall b \in B
\end{array}
$$

\section{Distillation Units}

Each distillation unit $d \in D$ is modeled with the following set of constraints:

Allocation constraints

The conditions that each distillation unit can be charged by at most one charging tank in a transfer event and at most one CDU can be charged by a single charging tank in a transfer event are enforced by eq (25) and eq (26) respectively.

$$
\begin{array}{ll}
\sum_{g \in G_{d}} w_{g d t} \leq 1 & \forall d \in D, \forall t \in T \\
\sum_{d \in D_{g}} w_{g d t} \leq 1 & \forall g \in G, \forall t \in T
\end{array}
$$

(ii) Continuous operation constraint

Each crude distillation unit (CDU) must be operated continuously and the total time of operation of each CDU must be equal to the time horizon $H$ (eq (27)). Because of the continuity required in the duration of operation, and the requirement that only one charging tank can 
charge a CDU over a period of time, for a CDU which is charged in transfer event $t$, the next charge (in transfer event $t+1$ ) will start at the ending time of the current transfer event $t$. This is enforced by eq (28) and eq (29).

$$
\begin{array}{ll}
\sum_{t} \sum_{g \in G_{d}}\left[T_{\text {gdt }}^{2}-T_{g d t}^{1}\right]=H & \forall d \in D \\
T_{g d, t+1}^{1} \geq T_{g^{\prime} d t}^{2}-H\left(1-w_{g^{\prime} d t}\right) & \forall g, g^{\prime} \in G_{d}, g \neq g^{\prime}, \forall d \in D, \forall t \in T, t<|T| \\
T_{g d, t+1}^{1} \leq T_{g^{\prime} d t}^{2}+H\left(1-w_{g^{\prime} d t}\right) & \forall g, g^{\prime} \in G_{d}, g \neq g^{\prime}, \forall d \in D, \forall t \in T, t<|T|
\end{array}
$$

\section{Supply Streams}

The supply streams have to follow certain mass balance and timing constraints:

(i) Timing Constraints

$$
\begin{aligned}
& T_{p}^{\text {start }} \leq T_{p s t}^{1}+H\left(1-w_{p s t}\right) \quad \forall p \in P, \forall s \in S_{p}, \forall t \in T \\
& T_{p}^{\text {end }} \geq T_{p s t}^{2}-H\left(1-w_{p s t}\right) \quad \forall p \in P, \forall s \in S_{p}, \forall t \in T
\end{aligned}
$$

These constraints state that all the flows from a supply stream $p$ to storage tank $s$ in any transfer event must start after a particular time $\left(T_{p}^{\text {start }}\right)$ and end before a certain time $\left(T_{p}^{\text {end }}\right)$. It is to be noted that the flow from a supply stream can be split such that one or more storage tanks are simultaneously fed by a single supply stream. Also, two or more suppply streams can feed the same storage tank at the same time.

(ii) Overall mass balances

The total amount of crude oil arriving in a supply stream $p$ (given by $V_{p}^{\text {supply }}$ ), must be completely transferred to the storage tanks over the set of all transfer events in the horizon.

$$
\sum_{t \in T} \sum_{s \in S_{p}} V_{p s t}^{\text {tot }}=V_{p}^{\text {supply }} \quad \forall p \in P
$$

(iii) Component balances 
The component flow from a supply stream $p$ to a tank $s$ (storage tank) in a transfer event $t$ is equal to the product of the total flow from that supply stream to the tank and the fraction of the component in the supply stream which is known.

$V_{j p s t}=f_{j p}^{\text {supply }} V_{p s t}^{\text {tot }} \quad \forall j \in J, \forall s \in S_{p}, \forall p \in P, \forall t \in T$

$f_{j p}^{\text {supply }}$ is the fraction of component $j$ in the supply stream $p$.

\section{Variable bounds}

All the continuous variables must lie between specified bounds and the discrete variables can be either 0 or 1 .

$$
\begin{aligned}
& 0 \leq I_{j b t} \leq I_{b}^{U} \quad \forall j \in J, \forall b \in B, \forall t \in T \\
& I_{b}^{L} \leq I_{b t}^{\text {tot }} \leq I_{b}^{U} \quad \forall b \in B, \forall t \in T \\
& 0 \leq V_{j a b t} \leq V_{a b}^{U} \quad \forall j \in J, \forall a \in A_{b}, \forall b \in B, \forall t \in T \\
& 0 \leq V_{j b c t} \leq V_{b c}^{U} \quad \forall j \in J, \forall c \in C_{b}, \forall b \in B, \forall t \in T \\
& 0 \leq V_{a b t}^{t o t} \leq V_{a b}^{U} \quad \forall a \in A_{b}, \forall b \in B, \forall t \in T \\
& 0 \leq V_{b c t}^{\text {tot }} \leq V_{b c}^{U} \quad \forall c \in C_{b}, \forall b \in B, \forall t \in T \\
& 0 \leq T_{a b t}^{1} \leq H \quad \forall a \in A_{b}, \forall b \in B, \forall t \in T \\
& 0 \leq T_{a b t}^{2} \leq H \quad \forall a \in A_{b}, \forall b \in B, \forall t \in T \\
& 0 \leq T_{b c t}^{1} \leq H \quad \forall c \in C_{b}, \forall b \in B, \forall t \in T \\
& 0 \leq T_{b c t}^{2} \leq H \quad \forall c \in C_{b}, \forall b \in B, \forall t \in T \\
& T_{p}^{\text {arr }} \leq T_{p}^{\text {start }} \leq H \quad \forall p \in P \\
& T_{p}^{\text {arr }} \leq T_{p}^{\text {end }} \leq H \quad \forall p \in P \\
& w_{a b t}, w_{b c t} \in\{0,1\}
\end{aligned}
$$

\section{Objective function}

The objective function used in this work is similar to the one used in Lee et al. (1996). 


$$
\begin{aligned}
\min \mathrm{z} & =\operatorname{Csea} \sum_{p \in P}\left(T_{p}^{\text {start }}-T_{p}^{\text {arrival }}\right)+\text { Cunload } \sum_{p \in P}\left(T_{p}^{\text {end }}-T_{p}^{\text {start }}\right)+ \\
& +H\left(\sum_{b \in B} \operatorname{Cinv}(b) \times\left(\sum_{t}\left(I_{b t}^{\text {tot }}+\sum_{a \in A_{b}} V_{a b t}^{\text {tot }}\right)+\sum_{t<|T|} I_{b t}^{\text {tot }}+2 I_{b}^{\text {init }- \text { tot }}\right)\right) /(2 \times N E+1) \\
+ & \operatorname{Cset}\left(\sum_{d \in D} \sum_{g \in G_{d}} \sum_{t} w_{g d t}-N D\right)
\end{aligned}
$$

where $\operatorname{Csea} \sum_{p}\left(T_{p}^{\text {start }}-T_{p}^{\text {arrival }}\right)$ is a waiting cost for a supply stream while the term Cunload $\sum_{p}\left(T_{p}^{\text {end }}-T_{p}^{\text {start }}\right)$ represents the unloading cost of crude for a supply stream. The total inventory maintenance cost of all the tanks in the system is given by the approximation $H\left(\sum_{b \in B} \operatorname{Cinv}(b) \times\left(\sum_{t}\left(I_{b t}^{t o t}+\sum_{a \in A_{b}} V_{a b t}^{t o t}\right)+\sum_{t<T \mid} I_{b t}^{t o t}+2 I_{b}^{\text {init-tot }}\right)\right) /(2 \times N E+1)$. This term is written in this way, since the model allows for both input into and output from a tank in the same transfer event, although they cannot be simultaneous. The last term $\operatorname{Cset}\left(\sum_{d \in D} \sum_{g \in G_{d}} \sum_{t} w_{g d t}-N D\right)$ corresponds to the setup cost of charging the ' $N D$ ' CDUs with different crude-mixes.

Equations (1) - (23), (25) - (34) comprise the MINLP model (P) which is to be optimized.

\section{Solution Strategy}

Large scale MINLPs such as problem (P) require specialized solution algorithms. We propose a specialized Outer-Approximation algorithm for solving the nonconvex model (P) to global optimality within a specified tolerance. In the proposed technique, we generate lower and upper bounds on the global optimum of (P) over a search region by solving separate models, which are then converged in the proposed algorithm.

\subsection{Lower Bounding problem}

A rigorous lower bound on the global optimum of problem $(\mathrm{P})$ can be obtained by solving an MILP relaxation of the original nonconvex MINLP model (P). This relaxation can be constructed by replacing the nonlinear equation (19) with eq (35) and using 
convex envelopes (see McCormick, 1976) (eqs (36) - (39)) for the bilinear terms appearing in eq (19), as given by the constraints below,

$$
\begin{array}{ll}
I_{j b c t}^{V J}+\sum_{a \in A_{b}} V_{j a b c t}^{V J}=I_{j b c t}^{V T}+\sum_{a \in A_{b}} V_{j a b c t}^{V T} & \forall j \in J, \forall t \in T, \forall c \in C_{b}, \forall b \in B \\
I_{j b c t}^{V J} \geq I_{b}^{L} V_{j b c t}+V_{b c}^{L} I_{b, t-1}^{t o t}-I_{b}^{L} V_{b c}^{L} & \forall j, \forall c \in C_{b}, \forall t \in T, \forall b \in B \\
I_{j b c t}^{V J} \geq I_{b}^{U} V_{j b c t}+V_{b c}^{U} I_{b, t-1}^{t o t}-I_{b}^{U} V_{b c}^{U} & \forall j, \forall c \in C_{b}, \forall t \in T, \forall b \in B \\
I_{j b c t}^{V J} \leq I_{b}^{L} V_{j b c t}+V_{b c}^{U} I_{b, t-1}^{t o t}-I_{b}^{L} V_{b c}^{U} & \forall j, \forall c \in C_{b}, \forall t \in T, \forall b \in B \\
I_{j b c t}^{V J} \leq I_{b}^{U} V_{j b c t}+V_{b c}^{L} I_{b, t-1}^{t o t}-I_{b}^{U} V_{b c}^{L} & \forall j, \forall c \in C_{b}, \forall t \in T, \forall b \in B
\end{array}
$$

$$
\begin{array}{ll}
I_{j b c t}^{V T} \geq I_{b}^{L} V_{b c t}^{t o t}+V_{b c}^{L} I_{j b, t-1}-I_{b}^{L} V_{b c}^{L} & \forall j, \forall c \in C_{b}, \forall t \in T, \forall b \in B \\
I_{j b c t}^{V T} \geq I_{b}^{U} V_{b c t}^{t o t}+V_{b c}^{U} I_{j b, t-1}-I_{b}^{U} V_{b c}^{U} & \forall j, \forall c \in C_{b}, \forall t \in T, \forall b \in B \\
I_{j b c t}^{V T} \leq I_{b}^{L} V_{b c t}^{t o t}+V_{b c}^{U} I_{j b, t-1}-I_{b}^{L} V_{b c}^{U} & \forall j, \forall c \in C_{b}, \forall t \in T, \forall b \in B \\
I_{j b c t}^{V T} \leq I_{b}^{U} V_{b c t}^{t o t}+V_{b c}^{L} I_{j b, t-1}-I_{b}^{U} V_{b c}^{L} & \forall j, \forall c \in C_{b}, \forall t \in T, \forall b \in B
\end{array}
$$

$$
\begin{array}{ll}
V_{j a b c t}^{V T} \geq V_{a b}^{L} V_{b c t}^{t o t}+V_{b c}^{L} V_{j a b t}-V_{a b}^{L} V_{b c}^{L} & \forall j, \forall a \in A_{b}, \forall c \in C_{b}, \forall t \in T, \forall b \in B \\
V_{j a b c t}^{V T} \geq V_{a b}^{U} V_{b c t}^{t o t}+V_{b c}^{U} V_{j a b t}-V_{a b}^{U} V_{b c}^{U} & \forall j, \forall a \in A_{b}, \forall c \in C_{b}, \forall t \in T, \forall b \in B \\
V_{j a b c t}^{V T} \leq V_{a b}^{L} V_{b c t}^{t o t}+V_{b c}^{U} V_{j a b t}-V_{a b}^{L} V_{b c}^{U} & \forall j, \forall a \in A_{b}, \forall c \in C_{b}, \forall t \in T, \forall b \in B \\
V_{j a b c t}^{V T} \leq V_{a b}^{U} V_{b c t}^{t o t}+V_{b c}^{L} V_{j a b t}-V_{a b}^{U} V_{b c}^{L} & \forall j, \forall a \in A_{b}, \forall c \in C_{b}, \forall t \in T, \forall b \in B
\end{array}
$$

The relaxed MILP problem (R) consists of eqs (1) - (18), (20) - (23), (25) - (39). The MILP relaxation (R) is often very large in size and requires significant computational effort to solve. To reduce the computational effort in solving this problem, we add cutting planes to model (R) which are derived using a technique, similar to that given in Karuppiah and Grossmann (2006). The description of the derivation of these cutting planes follows. The network is split into separate decoupled structures, as shown in Fig. 3, following the concept of spatial decomposition (e.g. see Jackson and Grossmann, 2003). Here the network is split into two decoupled sub-structures, although more sub- 
tructures are possible. The sub-structure to the left of the dotted line in Fig. 3, which is called D1 while the sub-structure on the right is termed D2. Physically, such a split can be interpreted as cutting the pipelines between some of the units in the network.

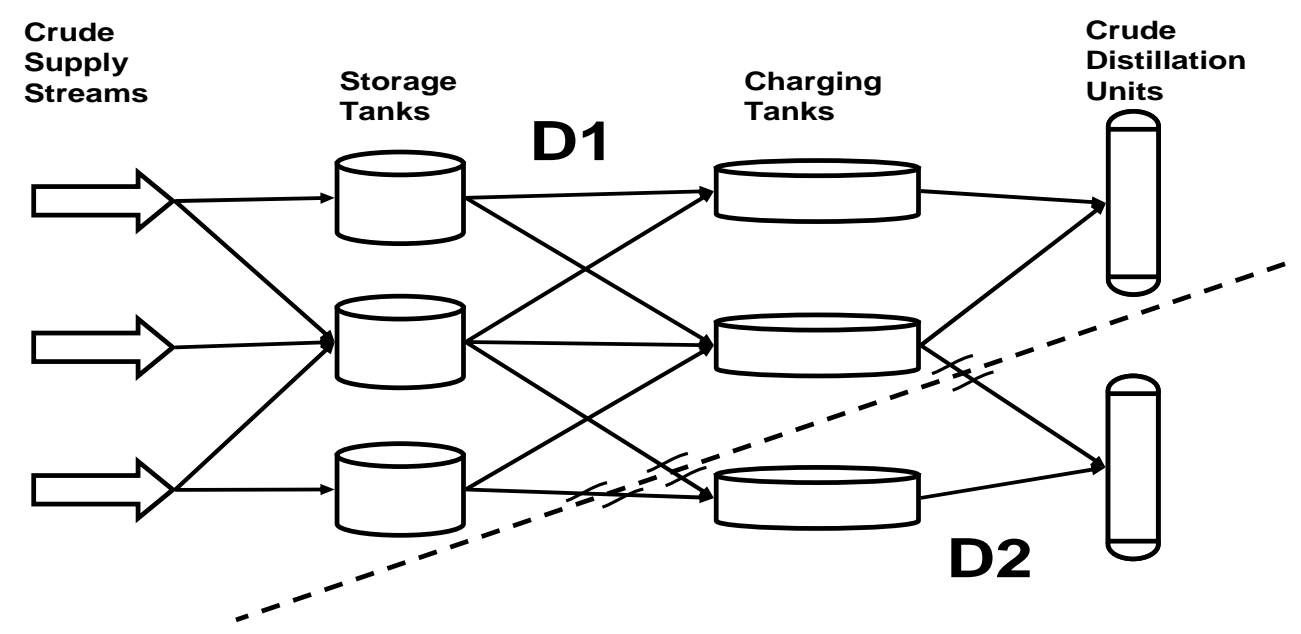

Fig. 3 Spatial decomposition of network structure

We then duplicate the variables pertaining to the flow existence (binary variables), total flow, component flows, and start and end times of flow for all the connections in the network that have been split. We end up with two sets of duplicate variables

$$
\left\{V_{m n t}^{t o t, 1}, V_{j m n t}^{1}(\forall j), T_{m n t}^{1,1}, T_{m n t}^{2,1}, w_{m n t}^{1}\right\} \quad \forall m \in M, \forall n \in N_{m}, \forall t
$$

and $\left\{V_{m n t}^{t o t, 2}, V_{j m n t}^{2}(\forall j), T_{m n n}^{1,2}, T_{m n t}^{2,2}, w_{m n t}^{2}\right\} \quad \forall m \in M, \forall n \in N_{m}, \forall t$, one set for each decomposed problem, and replace the variables $\left\{V_{m n t}^{\text {tot }}, V_{j m n t}(\forall j), T_{m n t}^{1}, T_{m n t}^{2}, w_{m n t}\right\} \quad \forall m \in M, \forall n \in N_{m}, \forall t$ with these newly created variables in model (P). The subscript $m$ stands for the source of the pipeline that has been split, while the subscript $n$ stands for the destination of a pipe that has been split. The variables $\left\{V_{m n t}^{t o t}, V_{j m n t}(\forall j), T_{m n t}^{1}, T_{m n t}^{2}, w_{m n t}\right\} \quad \forall m \in M, \forall n \in N_{m}, \forall t$ are said to be the linking variables since they link the different sub-structures. The remaining variables in model (P) are called non-linking variables since they are separate for both sub-structures D1 and D2. Due to the introduction of the duplicate variables, the equations involving the split pipelines get duplicated and are written in terms of the variables $\left\{V_{m n t}^{t o t, 1}, V_{j m n t}^{1}(\forall j), T_{m n t}^{1,1}, T_{m n t}^{2,1}, w_{m n t}^{1}\right\} \quad \forall m \in M, \forall n \in N_{m}, \forall t \quad$ (equations corresponding to D1) and $\left\{V_{m n t}^{t o t, 2}, V_{j m n t}^{2}(\forall j), T_{m n t}^{1,2}, T_{m n t}^{2,2}, w_{m n t}^{2}\right\} \quad \forall m \in M, \forall n \in N_{m}, \forall t$ (equations corresponding to D2). Further, since these newly formed variables are duplicates of the variables present in the original 
model, they are related by the following equality constraints which are added to model (P):

$$
\begin{array}{ll}
V_{m n t}^{t o t, 1}-V_{m n t}^{t o t, 2}=0 & \forall m \in M, \forall n \in N_{m}, \forall t \in T \\
V_{j m n t}^{1}-V_{j m n t}^{2}=0 & \forall j \in J, \forall m \in M, \forall n \in N_{m}, \forall t \in T \\
T_{m n t}^{1,1}-T_{m n t}^{1,2}=0 & \forall m \in M, \forall n \in N_{m}, \forall t \in T \\
T_{m n t}^{2,1}-T_{m n t}^{2,2}=0 & \forall m \in M, \forall n \in N_{m}, \forall t \in T \\
w_{m n t}^{1}-w_{m n t}^{2}=0 & \forall m \in M, \forall n \in N_{m}, \forall t \in T
\end{array}
$$

Equations (40) - (44) are then dualized, that is, they are multiplied by the Lagrange multipliers $\lambda_{m n t}^{V t o t}, \lambda_{j m n t}^{V C}(\forall j), \lambda_{m n t}^{T 1}, \lambda_{m n t}^{T 2}$ and $\lambda_{m n t}^{w} \quad \forall m \in M, \forall n \in N_{m}, \forall t$, respectively, and transferred to the objective function. This yields a Lagrangean relaxation of the original problem, which is denoted by (LRP), and is decomposable into smaller sub-problems corresponding to D1 and D2, which are easier to solve.

The model (LRP) is decomposed into two smaller sub-problems (LD1) and (LD2) such that model (LD1) includes equations and variables pertaining to structure D1, while model (LD2) includes equations and variables corresponding to the structure D2. The bounds of all the non-linking variables in both the sub-problems are the same as in the original full space problem (P). For the case of the duplicate variables, their bounds are the same as the bounds of the corresponding linking variables in the original problem. The two models (LD1) and (LD2) are as follows:

$$
\begin{aligned}
& \min z^{L D 1}=C \text { sea } \sum_{p \in P_{D 1}}\left(T_{p}^{\text {start }}-T_{p}^{\text {arrival }}\right)+\operatorname{Cunload} \sum_{p \in P_{D 1}}\left(T_{p}^{\text {end }}-T_{p}^{\text {start }}\right)+H\left(\sum_{b \in B_{D 1}} \operatorname{Cinv}_{b} \times\left(\sum_{t}\left(I_{b t}^{\text {tot }}+\sum_{a \in A_{b}} V_{a b t}^{\text {tot }}\right)+\sum_{t<T} I_{b t}^{\text {tot }}+2 I_{b}^{\text {init-tot }}\right)\right) /(2 \times N E+1) \\
& +\operatorname{Cset}\left(\sum_{d \in D_{D 1}} \sum_{g \in G_{d}} \sum_{t} w_{g d t}-N D_{D 1}\right)+ \\
& \sum_{m \in M} \sum_{n \in N_{m}} \sum_{t} \lambda_{m n t}^{V t o t} V_{m n t}^{t o t, 1}+\sum_{j} \sum_{m \in M} \sum_{n \in N_{m}} \sum_{t} \lambda_{j m n t}^{V C} V_{j m n t}^{1}+\sum_{m \in M} \sum_{n \in N_{m}} \sum_{t} \lambda_{m n t}^{T 1} T_{m n t}^{1,1}+\sum_{m \in M} \sum_{n \in N_{n}} \sum_{t} \lambda_{m n t}^{T 2} T_{m n t}^{2,1}+\sum_{m \in M} \sum_{n \in N_{m}} \sum_{t} \lambda_{m n t}^{w} t_{m n t}^{1} \\
& \text { s.t. constraints corresponding to units and connections in D1 }
\end{aligned}
$$




$$
\begin{aligned}
& \min z^{L D 2}=C \text { Sea } \sum_{p \in P_{D 2}}\left(T_{p}^{\text {start }}-T_{p}^{\text {arrival }}\right)+\text { Cunload } \sum_{p \in P_{D 2}}\left(T_{p}^{\text {end }}-T_{p}^{\text {start }}\right)+H\left(\sum_{b \in B_{D 2}} \operatorname{Cinv}_{b} \times\left(\sum_{t}\left(I_{b t}^{\text {tot }}+\sum_{a \in A_{b}} V_{a b t}^{\text {tot }}\right)+\sum_{t \in T T} I_{b t}^{\text {tot }}+2 I_{b}^{\text {init-tot }}\right)\right) /(2 \times N E+1) \\
& +\operatorname{Cset}\left(\sum_{d \in D_{D 2}} \sum_{g \in G_{d}} \sum_{t} w_{g d t}-N D_{D 2}\right)+
\end{aligned}
$$

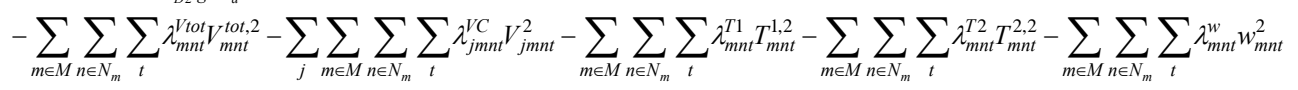

$$
\begin{aligned}
& \text { s.t. constrains correspondng to units and connectiors in D2 }
\end{aligned}
$$

The MILP relaxations of models (LD1) and (LD2), termed (LD1-R) and (LD2-R), respectively, are constructed by replacing the nonlinear terms in these models by convex envelopes. Models (LD1-R) and (LD2-R) are solved to obtain solutions $z_{1}^{*}$ and $z_{2}^{*}$, respectively. Using these solutions, the following valid linear cuts are generated in the full space of the original problem, which are given by eqs (45) and (46).

$$
\begin{aligned}
& z_{1}^{*} \leq C \operatorname{Csea} \sum_{p \in P_{D 1}}\left(T_{p}^{\text {start }}-T_{p}^{\text {arrivag }}\right)+\text { Cunload } \sum_{p \in P_{D 1}}\left(T_{p}^{\text {end }}-T_{p}^{\text {start }}\right)+H\left(\sum_{b \in B_{D 1}} \operatorname{Cinv} \times\left(\sum_{t}\left(I_{b t}^{\text {tot }}+\sum_{a \in A_{b}} V_{a b t}^{\text {tot }}\right)+\sum_{t \triangleleft T T} I_{b t}^{\text {tot }}+2 I_{b}^{\text {inittot }}\right)\right) /(2 \times N E+1) \\
& +\operatorname{Cse}\left(\sum_{d \in D_{D 1}} \sum_{g \in G_{d}} \sum_{t} w_{g d t}-N D_{D 1}\right)+ \\
& \sum_{m \in M} \sum_{n \in N_{m}} \sum_{t} \lambda_{m n}^{V t o t} V_{m n t}^{t o t}+\sum_{j} \sum_{m \in M} \sum_{n \in N_{m}} \sum_{t} \lambda_{j m n}^{V C} Y_{j m n t}+\sum_{m \in M} \sum_{n \in N_{m}} \sum_{t} \lambda_{m n t}^{T 1} T_{m n t}^{1}+\sum_{m \in M} \sum_{n \in N_{m}} \sum_{t} \lambda_{m n t}^{T 2} T_{m n t}^{2}+\sum_{m \in M} \sum_{n \in N_{m}} \sum_{t} \lambda_{m n}^{w}{ }^{w}{ }_{m n t}
\end{aligned}
$$

$$
\begin{aligned}
& z_{2}^{*} \leq C \operatorname{Csea} \sum_{p \in P_{D 2}}\left(T_{p}^{\text {start }}-T_{p}^{\text {arrivag }}\right)+\text { Cunload } \sum_{p \in P_{D 2}}\left(T_{p}^{\text {end }}-T_{p}^{\text {star }}\right)+H\left(\sum_{b \in B_{D 2}} \operatorname{Cing} \times\left(\sum_{t}\left(I_{b t}^{\text {tot }}+\sum_{a \in A_{b}} V_{a b t}^{\text {tot }}\right)+\sum_{t \triangleleft T \mid} I_{b t}^{\text {tot }}+2 I_{b}^{\text {init-tot }}\right)\right) /(2 \times N E+1) \\
& +C s e\left(\sum_{d \in D_{D 2}} \sum_{g \in G_{d}} \sum_{t} w_{g d t}-N D_{D 2}\right)+
\end{aligned}
$$

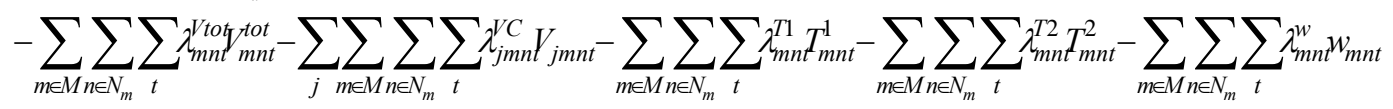

Theoretical properties of such cuts are given in Karuppiah and Grossmann (2006). Namely, the cuts are valid when added to the original problem, and the inclusion of the cuts into the relaxation $(\mathrm{R})$ produces a lower bound at least as strong as the lower bound obtained from Lagrangean decompsotion and the one obtained by solving (R) without any cuts. The Lagrange multipliers used in these cuts can be updated using a procedure given in the appendix, and additional cuts can be derived as described above. This procedure of updating the multiplers and adding cuts can be performed any number of 
times. It is important to note that the performance of these cuts in reducing the solution time of the relaxation strongly depends on the values of the Lagrange multipliers. The cuts (eqs (45) and/or (46)) are then added to (R) which is the MILP relaxation of model (P) to get a modified MILP model (RP). On solving (RP), we obtain a valid lower bound on the solution of $(\mathrm{P})$.

\subsection{Upper Bounding Sub-problem}

We fix the binary variables in problem $(\mathrm{P})$ to the values obtained from the solution of (RP), and obtain a nonconvex NLP model (P-NLP) which is solved to global optimality with any standard method. This then yields an upper bound on the solution of (P). The optimal values of the variables obtained from the solution of (RP) are then used as a starting point for the NLP solver. In case the model (P-NLP) is found to be infeasible for these integer values, we use as a heuristic to obtain alternate sub-optimal integer solutions by solving (RP) for an specified amount of time, and select the best found integer solution.

\subsection{Outer Approximation Algorithm}

The proposed Outer Approximation algorithm is shown in flowchart form in Fig. 4.

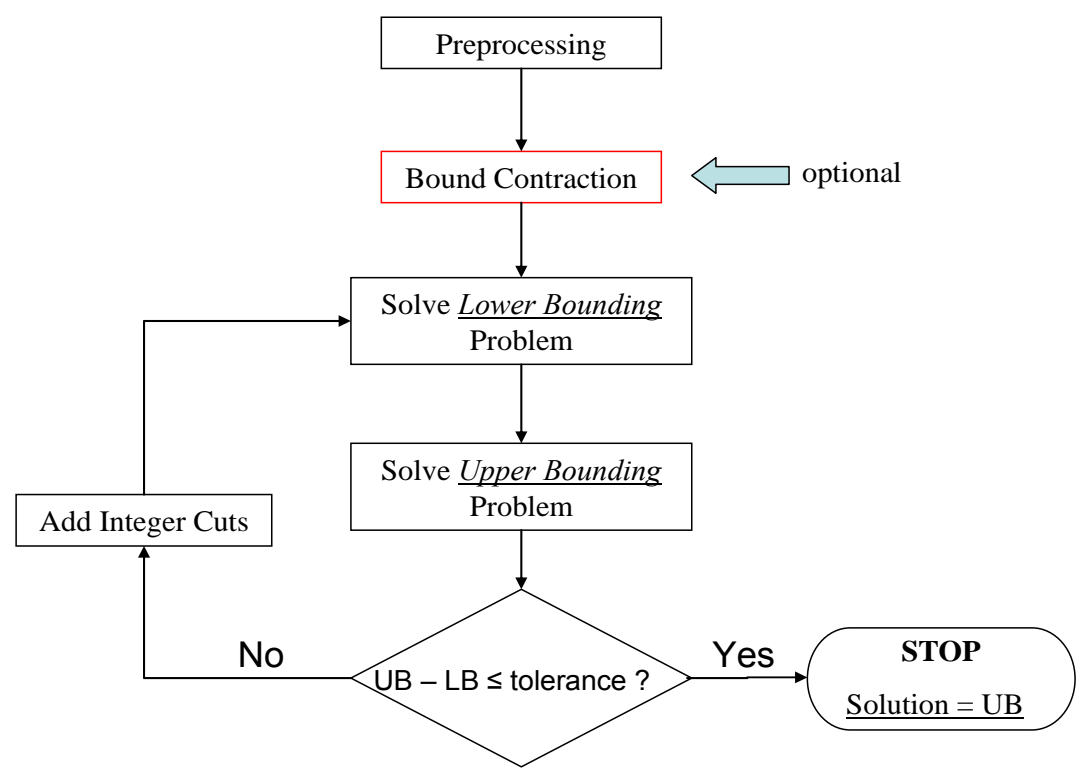

Fig. 4 Proposed Outer Approximation algorithm 
The algorithm is along the lines of the techniques proposed by Duran and Grossmann (1986), Kesavan et al. (2004), and Wei et al. (2005) and is outlined as follows:

a. Preprocessing The bounds of the variables in the model are determined by physical inspection of the network structure and using the numerical data given for the tanks, supply streams and the distillation units. Also, in this step, the original nonconvex MINLP may be locally optimized to obtain an initial overall upper bound (OUB) for the objective function.

b. Bound Contraction (Optional) The bounds of variables appearing in the nonconvex terms maybe contracted by solving a set of LPs using a procedure given in Zamora and Grossmann (1999), or the range reduction techniques in Tawarmalani and Sahinidis (2002).

c. Lower Bound Generation Generate a valid lower bound for the solution of the nonconvex MINLP following the technique outlined in section 4.1.

d. Upper Bound Generation Generate an upper bound using the method given in section 4.2 and update the OUB if the current upper bound is found to be better than the existing OUB.

e. Integer Cuts Using the integer solution obtained from solving (RP), add an integer cut to model (RP) to exclude this particular combination of binary variables. It is important to note that if the model (P-NLP) is not globally optimized in step $d$, adding these integer cuts to the relaxation in the next iteration could potentially cut off the global optimum.

f. Termination Iterate between solving models (RP) and (P-NLP) till the lower bound exceeds the upper bound or the relaxation gap between the lower and upper bounds is less than a specified tolerance. Convergence to the global optimum is not guaranteed if a local NLP solver is used in step $d$ above.

\section{Remarks}

(i) In a more traditional Lagrangean decomposition approach, the network is usually decomposed such that all the units present in it are separated. On solving the sub-models corresponding to every unit in the network, we obtain very weak cuts. To avoid this problem, we decompose the network into only 
two or three sub-structures. It is found heuristically that decomposing the network into unbalanced sub-structures and using cuts derived from the smaller sub-structures leads to a better performance of the algorithm.

(ii) There are multiple ways to split the network and generate sub-structures and corresponding cutting planes. For example, the structure shown in Fig. 1 can also be split into two sub-structures D3 and D4 as shown below in Fig. 5. Also the original network structure can be split into more than two sub-structures.

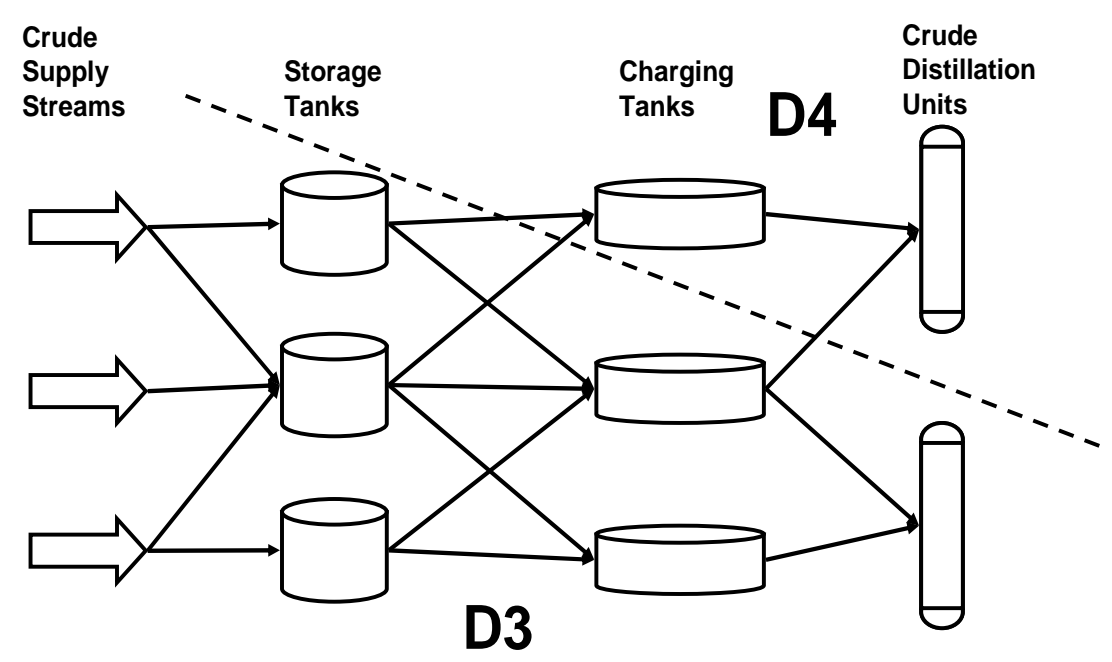

Fig. 5 Alternate decomposition scheme for network in Fig. 1

(iii) A proposed heuristic rule on how to split the original structure is as follows:

(a) Count the number of binary variables (nb) and constraints (nc) in the original MINLP model (P) pertaining to the optimization of the whole network structure.

(b) Pick the largest sub-structure resulting from the proposed split scheme and count the number of binary variables (nbs) and constraints (ncs) in the MINLP model corresponding to this sub-structure .

(c) Calculate the ratio : (nbs $+\mathrm{ncs}) /(\mathrm{nb}+\mathrm{nc})$ and check if it is less than 0.9. If this condition fails, the original structure has to be split differently into 
sub-structures such that (nbs $+\mathrm{ncs}) /(\mathrm{nb}+\mathrm{nc})$ for the largest sub-structure is below 0.9 .

\section{Examples}

The effectiveness of the proposed algorithm in solving scheduling problems is demonstrated using three examples for which the data is obtained from Lee et al. (1996). The units of some of the parameters are not specified in order to be consistent with the previous literature data. All examples were formulated using GAMS (Brooke et al., 1998) and solved on Intel 3.2 GHz Linux machine with 1024 MB memory. GAMS/CPLEX 9.0 was used for solving the MILP problems, while GAMS/CONOPT 3.0 and GAMS/BARON 7.2.5 were used for local optimization and global optimization, respectively, of the NLP problems. For comparison with the proposed algorithm, we also used GAMS/DICOPT and GAMS/BARON 7.2.5 for solving the MINLP models. Locally optimal solutions to the MINLP models are obtained using DICOPT (1 iteration for the relaxed NLP + 2 major iterations are performed) and compared against the solutions obtained from the proposed algorithm. Hence, the computational expense of solving the examples using DICOPT is not included in the total computational time taken by the algorithm. It is to be noted that when BARON (Sahinidis, 1996) was used to solve the NLP model (P-NLP), an optimality tolerance of $1 \%$ was used. The algorithm was terminated at the end of the first iteration for all the examples, since the relaxation gap between the lower and upper bound was sufficiently small within an acceptable tolerance for the global optimum. However, the iterations of the proposed Outer-Approximation algorithm may be continued to further reduce the gap between the lower and upper bounds. The problem sizes for all three examples is given in Table 4 and the various computational results are given in Tables $5 \mathrm{a}-5 \mathrm{~d}$. The number of transfer events was arbitrarily chosen to be 3 for all the units in all the examples, as the algorithm was able to find good solutions when the horizon was divided into 3 transfer events.

Example 1 The first example is a network consisting of 3 supply streams, 3 storage tanks, 3 charging tanks and 2 distillation units, whose structure is shown in Fig. 1. The crude oil 
in this example contains one key component and all the other components are combined into a bulk component, thus effectively making the given crude a two component system. The crude movement has to be scheduled over a time horizon of 12 hours. The relevant numerical data to carry out the optimization for this example is given in Table 1.

Table 1. Data for example 1

\begin{tabular}{|c|c|c|c|}
\hline $\begin{array}{c}\text { Scheduling Horizon (H) } \\
\text { sumber of crude supply } \\
\text { streams }\end{array}$ & \multicolumn{3}{|c|}{12 hours } \\
\hline $\begin{array}{c}\text { Crude Supply } \\
\text { Stream }\end{array}$ & $\begin{array}{c}\text { Arrival time } \\
\left(T_{p}^{a r r}\right)\end{array}$ & $\begin{array}{c}\text { Incoming } \\
\text { volume of crude }\end{array}$ & $\begin{array}{c}\text { Fraction of key } \\
\text { component }\end{array}$ \\
\hline IN1 & 1 & 50 & 0.01 \\
\hline IN2 & 5 & 50 & 0.085 \\
\hline IN3 & 9 & 50 & 0.06 \\
\hline
\end{tabular}

\begin{tabular}{|c|c|c|c|}
\hline \multicolumn{2}{|c|}{ Number of Storage Tanks } & \multicolumn{2}{c|}{3} \\
\hline $\begin{array}{c}\text { Storage } \\
\text { Tank }\end{array}$ & Capacity & Initial Oil Inventory & $\begin{array}{c}\text { Initial fraction of } \\
\text { key component (min - max) }\end{array}$ \\
\hline ST1 & 100 & 20 & $0.02(0.01-0.03)$ \\
\hline ST2 & 100 & 20 & $0.05(0.04-0.06)$ \\
\hline ST3 & 100 & 20 & $0.08(0.07-0.09)$ \\
\hline
\end{tabular}

\begin{tabular}{|c|c|c|c|}
\hline \multicolumn{2}{|c|}{ Number of Charging Tanks } & \multicolumn{2}{l|}{3} \\
\hline $\begin{array}{c}\text { Charging } \\
\text { Tank }\end{array}$ & Capacity & Initial Oil Inventory & $\begin{array}{c}\text { Initial fraction of } \\
\text { key component (min - max) }\end{array}$ \\
\hline CT1 & 100 & 30 & $0.03(0.025-0.035)$ \\
\hline CT2 & 100 & 50 & $0.05(0.045-0.065)$ \\
\hline CT3 & 100 & 30 & $0.08(0.075-0.085)$ \\
\hline
\end{tabular}


Number of CDUs : 2

Waiting cost for supply streams (Csea): 5

Unloading cost for supply streams (Cunload): 10

Tank inventory costs $\left(\operatorname{Cinv}_{b}\right)$ : storage tanks, 0.04; charging tanks, 0.08

Changeover cost for charged oil switch (Cset): 50

Demand of mixed oils by CDUs : oil mix 150

$$
\begin{array}{ll}
\text { oil mix } 2 & 50 \\
\text { oil mix } 3 & 50
\end{array}
$$

Bounds on flowrates in the streams: Lower Bound, 1; Upper Bound, 40

On applying the proposed algorithm to this example we obtain an optimal objective value of 282.19. The optimal crude schedule is shown in Fig. 6. The inventory profiles of the tanks are not given for this example and for the subsequent numerical examples since the model includes only the times when the crude transfers begin and end and there is no explicit information in the model pertaining to the start and end times of flow from a tank.

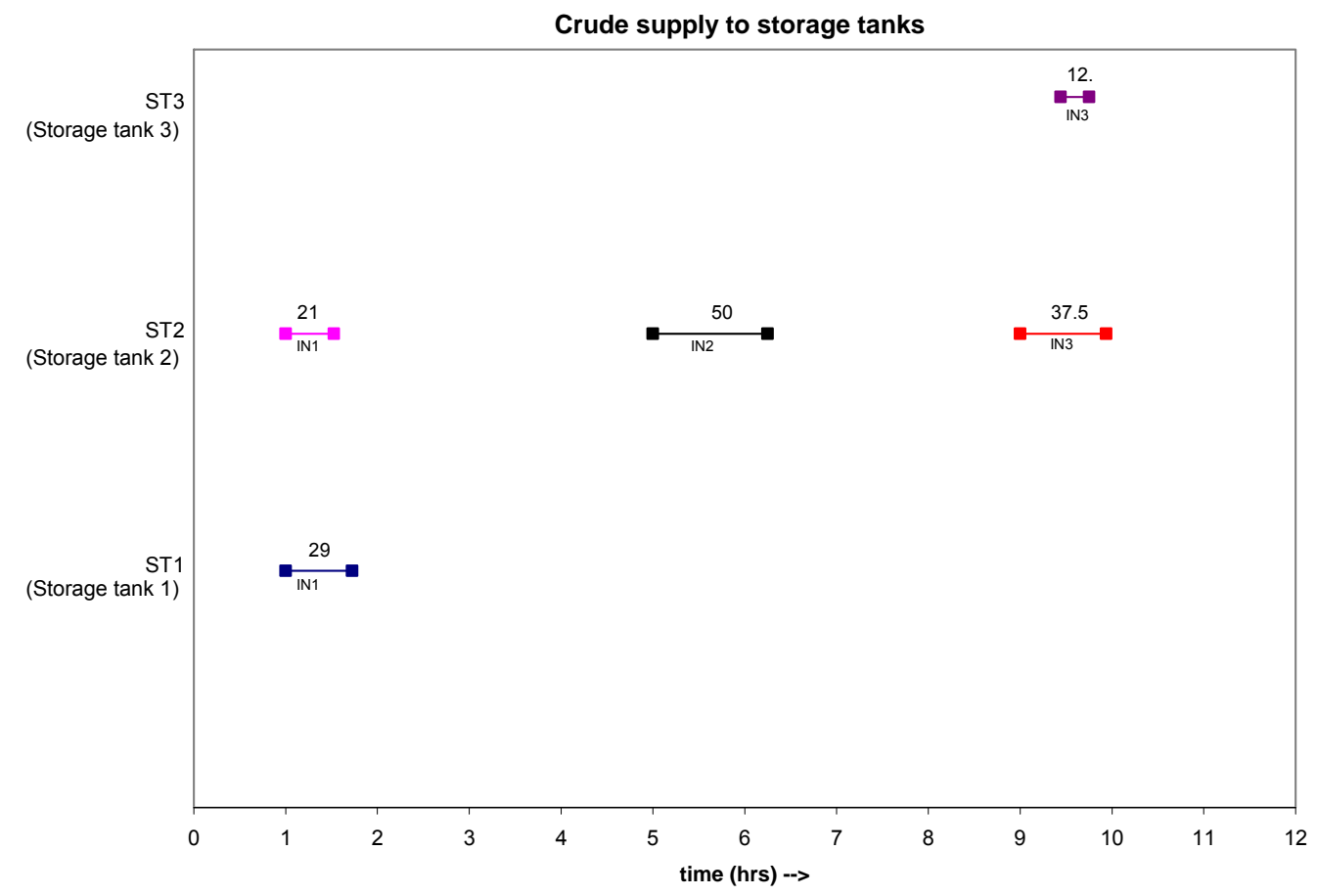



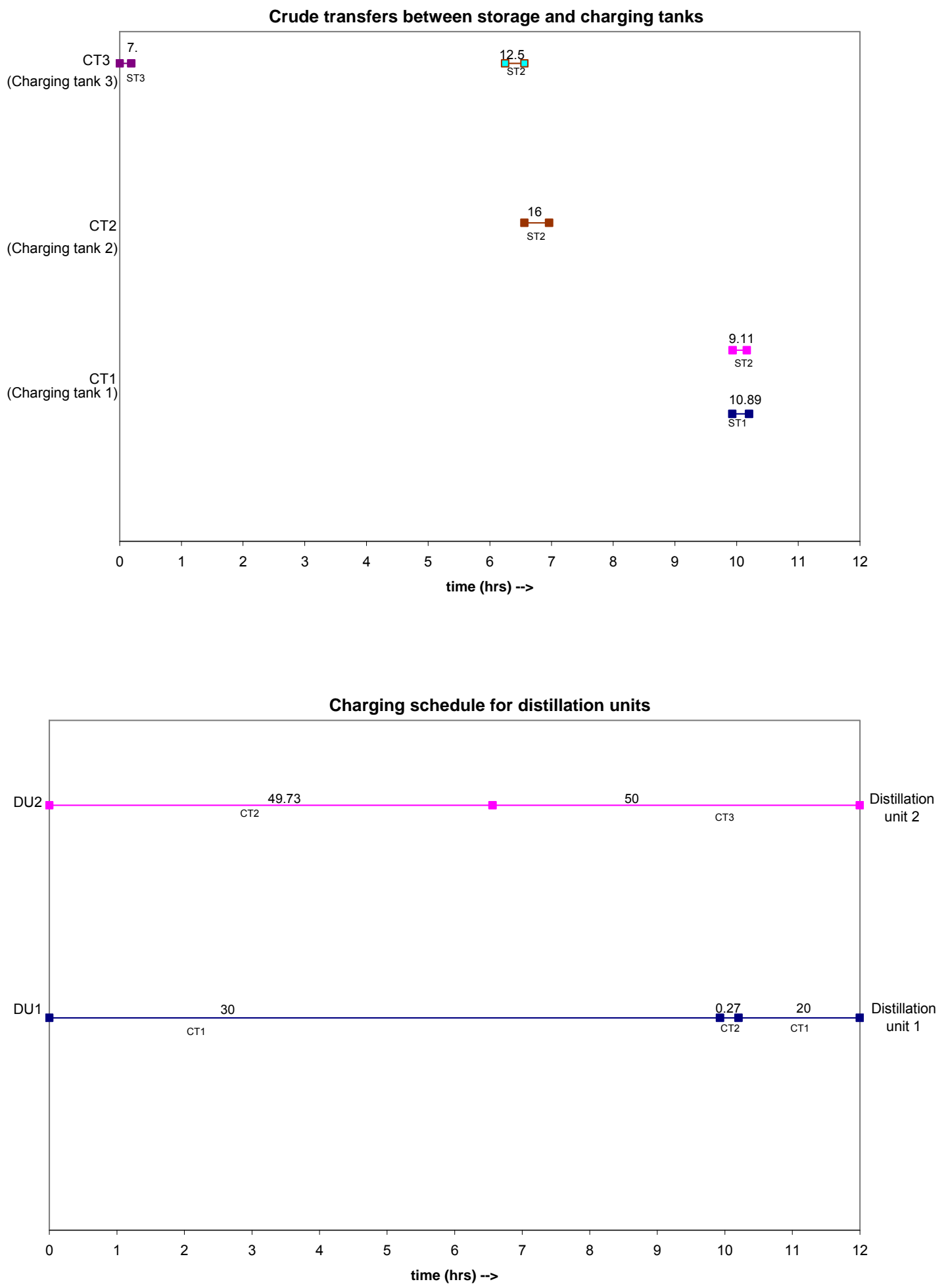

Fig. 6 Gantt chart of the schedule for example 1 
Example 2 The second example is very similar in structure to the first example and it also has 3 supply streams, 3 storage tanks, 3 charging tanks and 2 distillation units. The network structure is shown in Fig. 7. Here we have two key components in the crude oil instead of only one as in example 1. The crude in this example is hence a three component fluid with these key components along with the remaining bulk component. This makes the model size larger for this example.

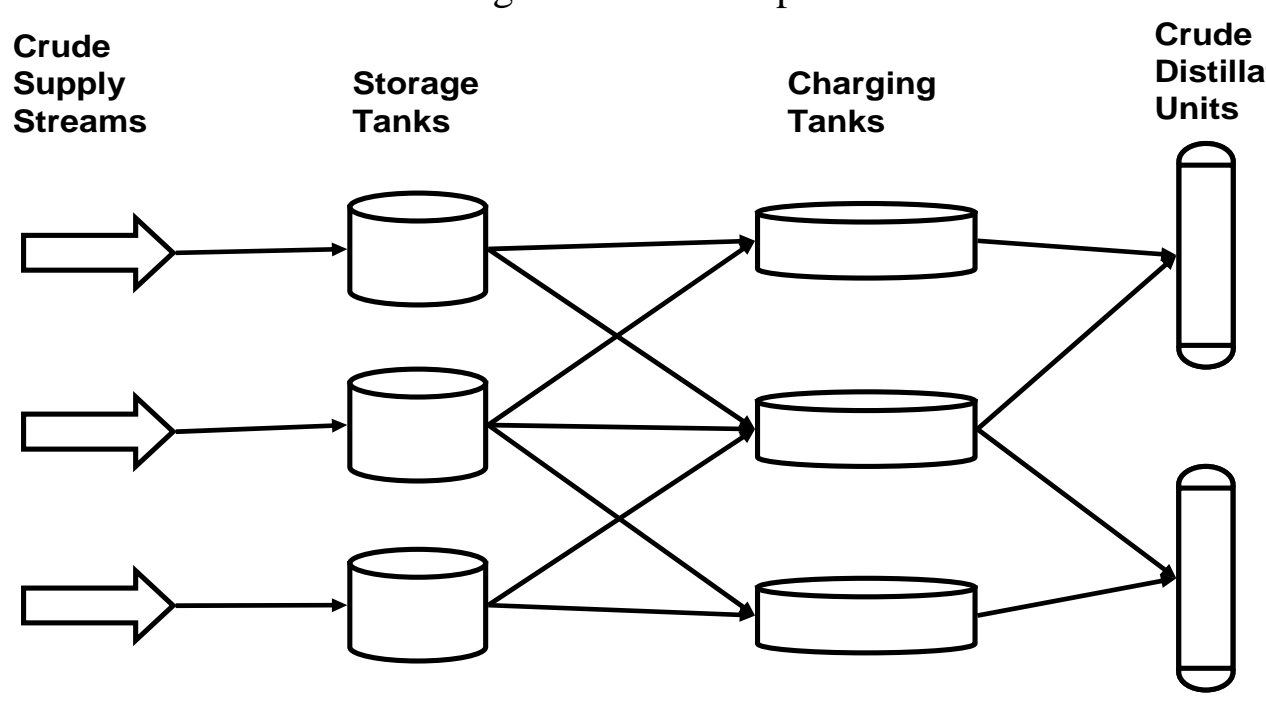

Fig. 7 Network structure for example 2

The scheduling has to be done for a time horizon of 10 hours. Table 2 provides the necessary data for the optimization and the optimal solution is given in Fig. 8.

Table 2. Data for example 2

\begin{tabular}{|c|c|c|c|c|}
\hline \multicolumn{2}{|c|}{$\begin{array}{l}\text { Scheduling Horizon (H) } \\
\text { streams }\end{array}$} & \multicolumn{4}{|c|}{3} \\
\hline $\begin{array}{c}\text { Crude Supply } \\
\text { Stream }\end{array}$ & $\begin{array}{c}\text { Arrival time } \\
\left(T_{p}^{\text {arr }}\right)\end{array}$ & $\begin{array}{c}\text { Incoming volume } \\
\text { of crude }\end{array}$ & $\begin{array}{c}\text { Fraction of } \\
\text { key } \\
\text { component } 1\end{array}$ & $\begin{array}{c}\text { Fraction of } \\
\text { key } \\
\text { component } 2\end{array}$ \\
\hline IN1 & 1 & 100 & 0.01 & 0.04 \\
\hline IN2 & 4 & 100 & 0.03 & 0.02 \\
\hline IN3 & 7 & 100 & 0.05 & 0.01 \\
\hline
\end{tabular}




\begin{tabular}{|c|c|c|c|c|}
\hline \multicolumn{2}{|c|}{ Number of Storage Tanks } & \multicolumn{3}{|c|}{3} \\
\hline $\begin{array}{c}\text { Storage } \\
\text { Tank }\end{array}$ & Capacity & $\begin{array}{c}\text { Initial Oil } \\
\text { Inventory }\end{array}$ & $\begin{array}{c}\text { Initial fraction of key } \\
\text { component } 1\end{array}$ & $\begin{array}{c}\text { Initial fraction of key } \\
\text { component 2 }\end{array}$ \\
\hline ST1 & 100 & 20 & 0.01 & 0.04 \\
\hline ST2 & 100 & 50 & 0.03 & 0.02 \\
\hline ST3 & 100 & 70 & 0.05 & 0.01 \\
\hline
\end{tabular}

\begin{tabular}{|c|c|c|c|c|}
\hline \multicolumn{2}{|c|}{ Number of Charging Tanks } & \multicolumn{3}{|c|}{3} \\
\hline $\begin{array}{c}\text { Charging } \\
\text { Tank }\end{array}$ & Capacity & $\begin{array}{c}\text { Initial Oil } \\
\text { Inventory }\end{array}$ & $\begin{array}{c}\text { Initial fraction of key } \\
\text { component } 1 \\
(\min -\max )\end{array}$ & $\begin{array}{c}\text { Initial fraction of key } \\
\text { component } 2 \\
(\min -\max )\end{array}$ \\
\hline CT1 & 100 & 30 & $0.0167(0.01-0.02)$ & $0.0333(0.03-0.038)$ \\
\hline CT2 & 100 & 50 & $0.03(0.025-0.035)$ & $0.023(0.018-0.027)$ \\
\hline CT3 & 100 & 30 & $0.0433(0.04-0.048)$ & $0.0133(0.01-0.018)$ \\
\hline
\end{tabular}

Number of CDUs : $\quad 3$

Waiting cost for supply streams (Csea): 5

Unloading cost for supply streams (Cunload): 8

Tank inventory costs $\left(\operatorname{Cinv}_{b}\right)$ : storage tanks, 0.05 ; charging tanks, 0.08

Changeover cost for charged oil switch (Cset): 30

Demand of mixed oils by CDUs : oil mix 1100

oil mix $2 \quad 100$

oil mix $3 \quad 100$

Bounds on flowrates in the streams: Lower Bound, 1; Upper Bound, 40 

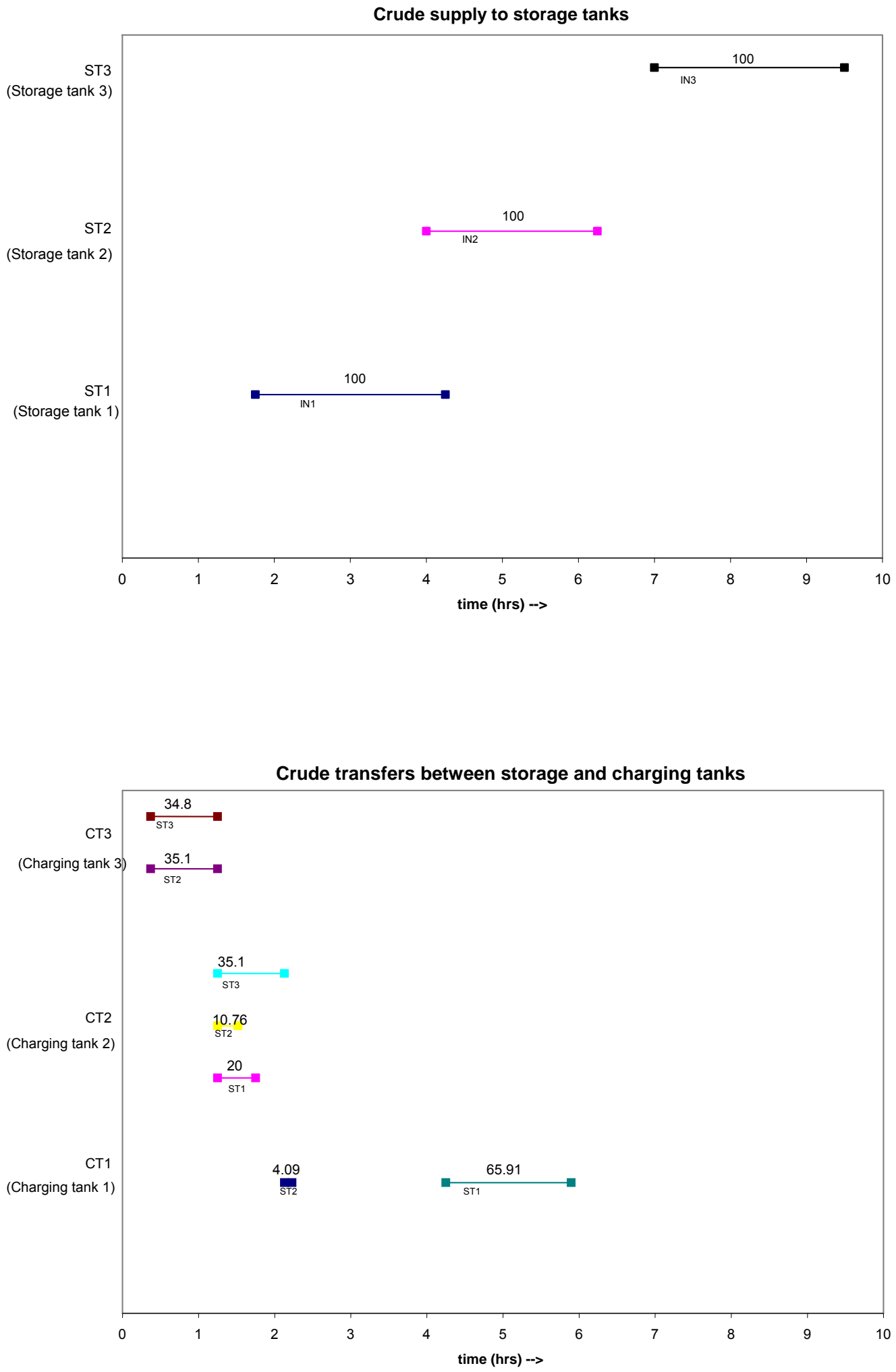


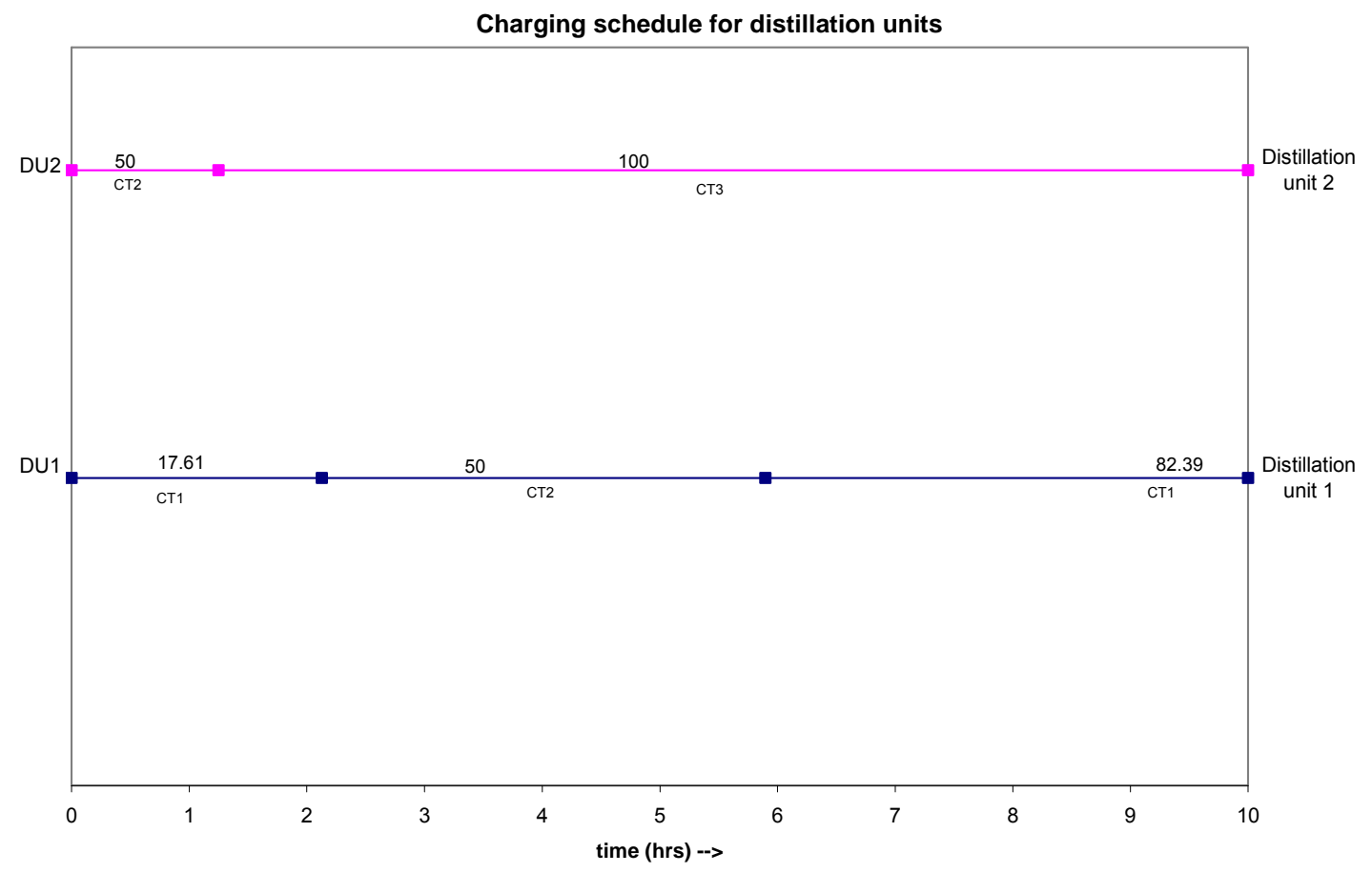

Fig. 8 Optimal solution for example 2

Example 3 The final example is an industrial size problem with 3 supply streams, 6 storage tanks, 4 charging tanks and 3 distillation units. The crude oil has to be scheduled over a time horizon of 15 hours. The crude oil in this system involves a single key component as in example 1 and other components combined into a bulk component. The network structure is shown in Fig. 9. 


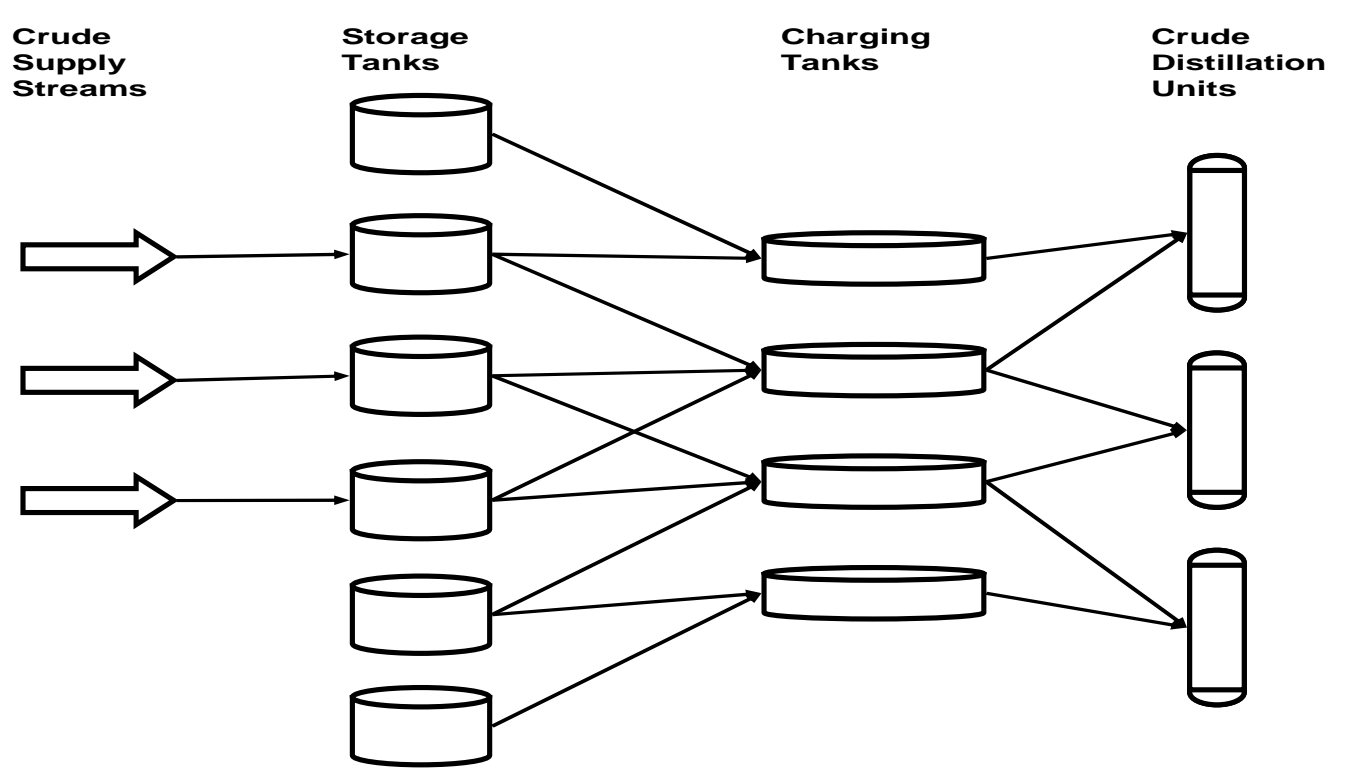

Fig. 9 Network structure for example 3

The relevant numerical data for this example is given in Table 3 while the optimal schedule is shown in Fig. 10.

Table 3. Data for example 3

\begin{tabular}{|c|c|c|c|}
\hline \multicolumn{2}{|c|}{$\begin{array}{c}\text { Scheduling Horizon (H) } \\
\text { streams }\end{array}$} & \multicolumn{3}{|c|}{3 hours } \\
\hline $\begin{array}{c}\text { Number of crude supply } \\
\text { Crude Supply } \\
\text { Stream }\end{array}$ & $\begin{array}{c}\text { Arrival Time } \\
\left(T_{p}^{\text {arr }}\right)\end{array}$ & $\begin{array}{c}\text { Incoming } \\
\text { Volume of } \\
\text { crude }\end{array}$ & $\begin{array}{c}\text { Fraction of key } \\
\text { component }\end{array}$ \\
\hline IN1 & 1 & 60 & 0.03 \\
\hline IN2 & 6 & 60 & 0.05 \\
\hline IN3 & 11 & 60 & 0.065 \\
\hline
\end{tabular}

\begin{tabular}{|c|c|c|c|}
\hline \multicolumn{2}{|c|}{ Number of Storage Tanks } & \multicolumn{2}{c|}{6} \\
\hline $\begin{array}{c}\text { Storage } \\
\text { Tank }\end{array}$ & Capacity & Initial Inventory & $\begin{array}{c}\text { Initial fraction of } \\
\text { key component (min - max) }\end{array}$ \\
\hline ST1 & $10-90$ & 60 & $0.031(0.025-0.038)$ \\
\hline ST2 & $10-110$ & 10 & $0.03(0.02-0.04)$ \\
\hline
\end{tabular}




\begin{tabular}{|l|c|c|c|}
\hline ST3 & $10-110$ & 50 & $0.05(0.04-0.06)$ \\
\hline ST4 & $10-110$ & 40 & $0.065(0.06-0.07)$ \\
\hline ST5 & $10-90$ & 30 & $0.075(0.07-0.08)$ \\
\hline ST6 & $10-90$ & 60 & $0.075(0.07-0.08)$ \\
\hline
\end{tabular}

\begin{tabular}{|c|c|c|c|}
\hline \multicolumn{2}{|c|}{ Number of Charging Tanks } & \multicolumn{2}{c|}{4} \\
\hline $\begin{array}{c}\text { Charging } \\
\text { Tank }\end{array}$ & Capacity & Initial Inventory & $\begin{array}{c}\text { Initial Fraction of } \\
\text { key component (min - max) }\end{array}$ \\
\hline CT1 & 80 & 5 & $0.0317(0.03-0.035)$ \\
\hline CT2 & 80 & 30 & $0.0483(0.043-0.05)$ \\
\hline CT3 & 80 & 30 & $0.0633(0.06-0.065)$ \\
\hline CT4 & 80 & 30 & $0.075(0.071-0.08)$ \\
\hline
\end{tabular}

Number of CDUs : $\quad 3$

Waiting cost for supply streams (Csea): 5

Unloading cost for supply streams (Cunload): 7

Tank inventory costs $\left(\operatorname{Cinv}_{b}\right)$ : storage tanks, 0.05 ; charging tanks, 0.06

Changeover cost for charged oil switch (Cset): 30

Demand of mixed oils by CDUs : oil mix 160

$\begin{array}{cc}\text { oil mix } 2 & 60 \\ \text { oil mix } 3 & 60 \\ \text { oil mix } 4 & 60\end{array}$

Bounds on flowrates in the streams: Lower Bound, 1; Upper Bound, 40 

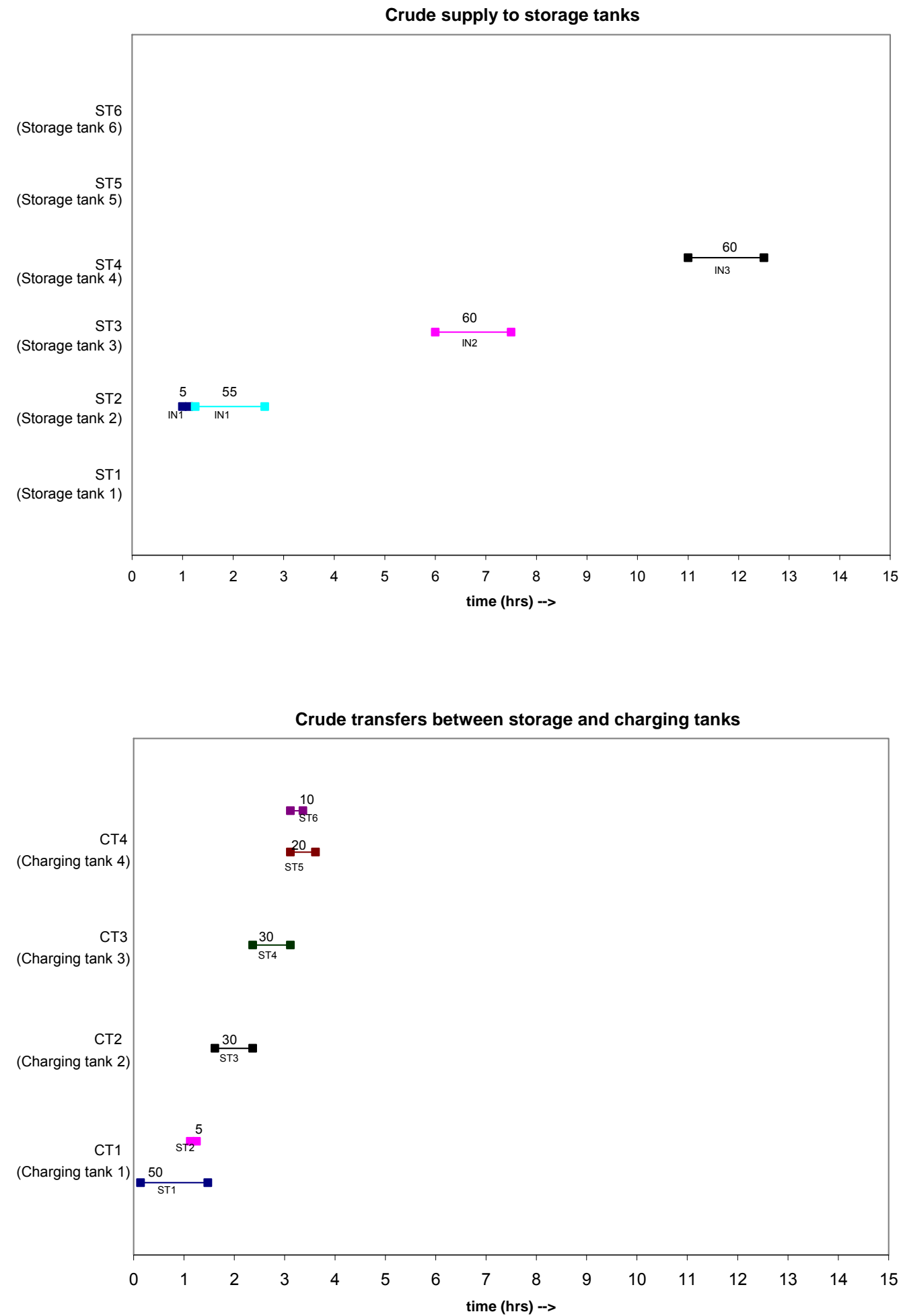


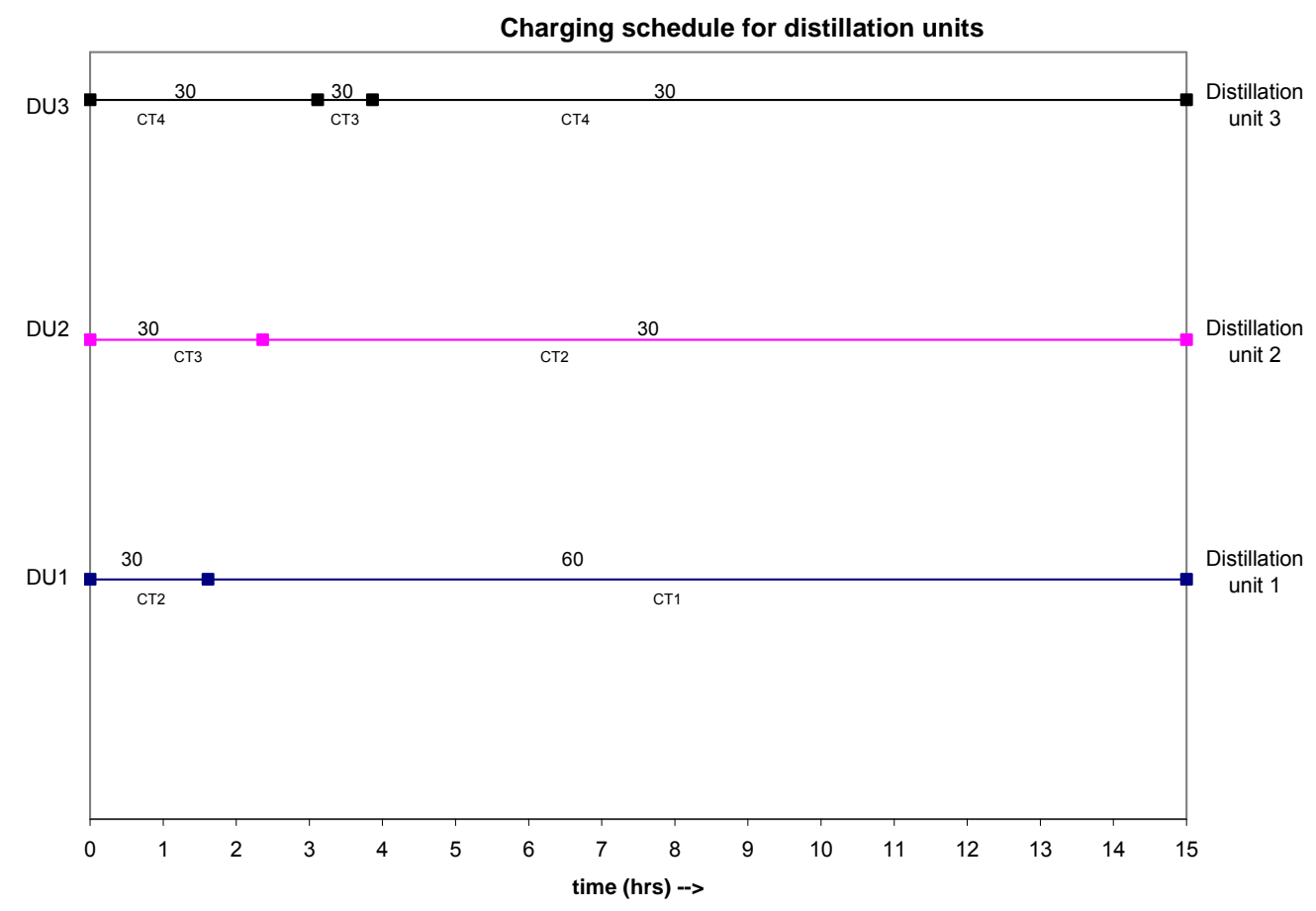

\section{Fig. 10 Optimal schedule for example 3}

On solving the problem to optimality using the proposed algorithm, we get a solution of 383.69, which is globally optimal within a tolerance of $1 \%$.

\section{Computational results}

The model sizes for the different examples are shown in Table 4. The new formulation is quite efficient for these crude oil scheduling problems and we obtain good solutions to the scheduling problem at the very first iteration of the proposed algorithm as seen in Table 5a. The algorithm finds the optima and proves their global optimality in tractable computational times. The addition of cutting planes to the MILP relaxation (R) as described in the algorithm decreases the number of nodes in the branch and bound tree for solving the MILP relaxation, and hence the solution time for solving the MILP. This is evident from Table 5b. The cuts added to the relaxation (R) tighten the lower bound at the root node of the branch and bound tree for the MILP, which contributes to reducing the number of nodes in the tree to about one third for solving the MILP.

For all the examples, the network structure is split into D1 and D2 (Fig. 2) and also into D3 and D4 (Fig. 3). The information about the sub-structures (D1, D2, D3 and 
D4) for example 2 and example 3 can be obtained from the authors. Initially a set of Lagrange multipliers was chosen to generate cuts (see Table $5 \mathrm{c}$ for multiplier information), and they are updated once more to generate more cuts, which are added to a pool of cuts. The column under the heading $z^{L}$ in this table gives the value of the lower bound on the global optimum of (P) obtained by conventional Lagrangean decomposition using the initially chosen set of Lagrange multipliers. We can see from Table $5 b$ that they are weaker than the lower bound obtained by solving the MILP relaxation (R) to optimality. From this pool of cuts that is generated, the weaker cuts derived from the smaller sub-structures, are chosen to be added to model (R) for all the numerical examples in the paper and model (R) is then solved. The solution of $(R)$ is used to fix the binary variables in model (P), and we solve the resulting NLP model to global optimality using BARON to get a solution. Here, we should note that BARON cannot solve the original MINLP problems (P) to global optimality even after 10 hours of CPU time.

At the end, we also tested the effectiveness of adding the proposed cuts to the MINLP model (P) corresponding to all examples. It is found that BARON performs better when trying to solve the MINLP model (P) with these cuts added to it, than it does in absence of these cuts. It can be seen from Table $5 d$ that the lower and upper bounds found by BARON at the end of 10 hours of computation, are closer to each other in the presence of these cuts, although the relaxation gap is still rather substantial.

Table 4. Model sizes for examples 1 - 3

\begin{tabular}{|c|c|c|c|}
\hline \multirow{2}{*}{ Example } & \multicolumn{3}{|c|}{ Original MINLP model (P) } \\
\cline { 2 - 4 } & $\begin{array}{c}\text { Number of } \\
\text { Binary } \\
\text { Variables }\end{array}$ & $\begin{array}{c}\text { Number of } \\
\text { Continuous } \\
\text { Variables }\end{array}$ & $\begin{array}{c}\text { Number of } \\
\text { Constraints }\end{array}$ \\
\hline 1 & 48 & 300 & 946 \\
\hline 2 & 42 & 330 & 994 \\
\hline 3 & 57 & 381 & 1167 \\
\hline
\end{tabular}


Table 5a. Numerical results for test examples 1 - 3

\begin{tabular}{|c|c|c|c|c|c|}
\hline Example & $\begin{array}{c}\text { Lower bound } \\
\text { [obtained by solving } \\
\text { relaxation (RP) ] } \\
\left(z^{R P}\right)\end{array}$ & $\begin{array}{c}\text { Upper bound } \\
\text { [on solving } \\
\text { (P-NLP) using } \\
\text { BARON ] } \\
\left(z^{P-N L P}\right)\end{array}$ & $\begin{array}{c}\text { Relaxation } \\
\text { gap (\%) }\end{array}$ & $\begin{array}{c}\text { Total time } \\
\text { taken for one } \\
\text { iteration }{ }^{\dagger} \text { of } \\
\text { algorithm } \\
\text { (CPUsecs) }\end{array}$ & $\begin{array}{c}\text { Local optimum } \\
\text { (using } \\
\text { DICOPT) }\end{array}$ \\
\hline 1 & 281.14 & 282.19 & 0.37 & 827.7 & 291.93 \\
\hline 2 & 351.32 & 359.48 & 2.27 & 6913.9 & 361.63 \\
\hline 3 & 383.69 & 383.69 & 0 & 8928.6 & 383.69 \\
\hline
\end{tabular}

Table 5b. Comparison of relaxations with and without cuts

\begin{tabular}{|c|c|c|c|c|c|c|c|c|}
\hline \multirow{2}{*}{ Example } & \multicolumn{4}{|c|}{ Solving MILP model (R) } & \multicolumn{3}{c|}{ Solving MILP model (RP) } \\
& \multicolumn{3}{|c|}{$\begin{array}{c}\text { LP } \\
\text { Solution } \\
\left(z^{R}\right)\end{array}$} & $\begin{array}{c}\text { including proposed cuts) } \\
\text { relaxation } \\
\text { at root } \\
\text { node }\end{array}$ & $\begin{array}{c}\text { No. of } \\
\text { nodes } \\
\text { noken to } \\
\text { solve (R) } \\
\text { (CPUsecs) }\end{array}$ & $\begin{array}{c}\text { Solution } \\
\left(z^{R P}\right)\end{array}$ & $\begin{array}{c}\text { LP } \\
\text { relaxation } \\
\text { at root } \\
\text { node }\end{array}$ & $\begin{array}{c}\text { No. of } \\
\text { nodes } \\
\text { taken to } \\
\text { solve (RP) } \\
\text { (CPUsecs) }\end{array}$ \\
\hline 1 & 281.14 & -55.24 & 940800 & 1953.3 & 281.14 & 68.45 & 334300 & 758.8 \\
\hline 2 & 351.32 & 113.35 & 931700 & 14481.7 & 351.32 & 133.80 & 310600 & 5873.2 \\
\hline 3 & 383.69 & 147.24 & 3029600 & 15874.8 & 383.69 & 189.19 & 1258100 & 8025.9 \\
\hline
\end{tabular}

${ }^{\dagger}$ Total time includes time for generating a pool of cuts, updating Lagrange multipliers, solving the relaxation (RP) using CPLEX and solving (P-NLP) using BARON 


\section{Table 5c. Lagrange multiplier information}

\begin{tabular}{|c|c|c|c|c|c|c|c|c|c|}
\hline \multirow{2}{*}{ Example } & \multirow{2}{*}{$\begin{array}{c}\text { Decomposition } \\
\text { scheme }\end{array}$} & \multicolumn{5}{|c|}{ Initial values of Lagrange multipliers } & \multirow{2}{*}{$z^{U}$} & \multirow{2}{*}{$z^{L}=z_{1}^{*}+z_{2}^{*}$} & \multirow{2}{*}{$\alpha$} \\
\hline & & $\lambda_{m n t}^{V t o t}$ & $\lambda_{j m n t}^{V C}$ & $\lambda_{m n t}^{T 1}$ & $\lambda_{m n t}^{T 2}$ & $\lambda_{m n t}^{w}$ & & & \\
\hline \multirow{2}{*}{1} & D1 and D2 & 0 & 0 & 0 & 0 & 0 & 282.225 & 201.335 & 0.4 \\
\hline & D3 and D4 & 0.01 & 0.01 & 0.01 & 0.01 & 0.01 & 282.225 & 202.324 & 0.4 \\
\hline \multirow{2}{*}{2} & D1 and D2 & 0 & 0 & 0 & 0 & 0 & 357.892 & 276.582 & 0.4 \\
\hline & D3 and D4 & 0.01 & 0.01 & 0.01 & 0.01 & 0.01 & 358.892 & 279.99 & 0.4 \\
\hline \multirow{2}{*}{ 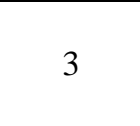 } & D1 and D2 & 0 & 0 & 0 & 0 & 0 & 393.157 & 344.267 & 0.4 \\
\hline & D3 and D4 & 0 & 0 & 0 & 0 & 0 & 393.157 & 348.214 & 0.5 \\
\hline
\end{tabular}

Table 5d. Effect of cuts on the performance of BARON in optimizing (P)

\begin{tabular}{|c|c|c|c|c|}
\hline \multirow{2}{*}{ Example } & \multicolumn{2}{|c|}{$\begin{array}{c}\text { Optimizing (P) using BARON } \\
\text { (without cuts) }\end{array}$} & \multicolumn{2}{c|}{$\begin{array}{c}\text { Optimizing (P) using BARON } \\
\text { (with cuts) }\end{array}$} \\
\cline { 2 - 5 } & Upper Bound (UB) & Lower Bound (LB) & Upper Bound (UB) & Lower Bound (LB) \\
\hline 1 & 282.19 & 32.62 & 282.19 & 75.31 \\
\hline 2 & 361.63 & 185.75 & 361.63 & 194.98 \\
\hline 3 & 383.69 & 214.07 & 383.69 & 233.30 \\
\hline
\end{tabular}

\section{Conclusions}

In this work, we have developed improved techniques for global optimization of scheduling the flow of crude oil at the front-end of a refinery. A continuous time model based on transfer events is used to represent the scheduling problem and this model is a nonconvex MINLP model which has multiple local optima. In order to obtain provably globally optimal solutions to the problem, we propose a specialized Outer-Approximation algorithm. In this, we generate lower and upper bounds on the global optimum which are

\footnotetext{
${ }^{\ddagger}$ BARON was run for a total time of 10 CPUhours
} 
converged to a specified tolerance. A rigorous lower bound on the global optimum is obtained by solving a MILP relaxation of the original problem. To reduce the computational effort required in solving this MILP relaxation, cutting planes derived from a spatial decomposition of the network are added to the MILP model. The solution of the MILP is used in a heuristic to obtain a feasible solution to the MINLP, which serves as an upper bound. The application of the proposed algorithm on different examples helps in significantly reducing the computational effort involved in solving such problems.

\section{Acknowledgments}

The authors gratefully acknowledge financial support from the National Science Foundation under Grant CTS-0521769 and from ExxonMobil Research and Engineering.

\section{Nomenclature}

\section{Indices}

a tanks input source

b crude tank

C tank output destination

d distillation unit

g charging tank

j component

$\mathrm{k} \quad$ node in the network

m source unit of split pipline

n destination unit of split pipeline

p supply stream

S storage tank

t transfer event

Sets

A Set of tanks input sources 

$A_{\mathrm{b}} \quad$ Set of inputs to a tank $b$
$\mathrm{A}_{\mathrm{s}} \quad$ Set of inputs to storage tank $s$
B Set of tanks
$\mathrm{B}_{\mathrm{D} 1} \quad$ Set of tanks belonging to sub-structure D1
$\mathrm{B}_{\mathrm{D} 2} \quad$ Set of tanks belonging to sub-structure D2
C Set of tank output destinations
$\mathrm{C}_{\mathrm{b}} \quad$ Set of outputs from a $\operatorname{tank} b$
$\mathrm{C}_{\mathrm{s}} \quad$ Set of outputs from storage tank $s$
$\mathrm{C}_{\mathrm{g}} \quad$ Set of outputs from charging tank $g$
D Set of distillation units
$\mathrm{D}_{\mathrm{D} 1} \quad$ Set of distillation units present in structure D1
$\mathrm{D}_{\mathrm{D} 2} \quad$ Set of distillation units present in structure D2
$\mathrm{D}_{\mathrm{g}} \quad$ Set of distillation units that can be charged by charging tank $g$
G(B) Set of charging tanks
$\mathrm{G}_{\mathrm{d}} \quad$ Set of charging tanks that charge distillation unit $d$
$\mathrm{J} \quad$ Set of components
$\mathrm{K} \quad$ Set of nodes in the network
M Set of source units of the split pipelines
$\mathrm{N}_{\mathrm{m}} \quad$ Set of destination units of split pipelines with source $m$
$\mathrm{P} \quad$ Set of supply streams
$\mathrm{P}_{\mathrm{D} 1} \quad$ Set of supply streams present in sub-structure D1
$\mathrm{P}_{\mathrm{D} 2} \quad$ Set of supply streams present in sub-structure D2
S(B) Set of storage tanks
$\mathrm{S}_{\mathrm{p}} \quad$ Set of storage tanks connected to supply stream $p$
$\mathrm{T} \quad$ Set of transfer events

\section{Parameters}

$\operatorname{Cinv}_{b} \quad$ Inventory maintenance cost for tank $b$

Csea Waiting cost for supply streams

Cset Changeover cost for charged oil switch

Cunload Unloading cost for supply streams 
$F_{a b}^{U} \quad$ Upper bound on flowrate from $a$ to $b$

$F_{a b}^{L} \quad$ Lower bound on flowrate from $a$ to $b$

$f_{j b}^{L} \quad$ Lower bound on fraction of component $j$ inside tank $b$

$f_{j b}^{U} \quad$ Upper bound on fraction of component $j$ inside tank $b$

$f_{j p}^{\text {supply }} \quad$ Fraction of component $j$ in supply stream $p$

$H \quad$ Time horizon for scheduling

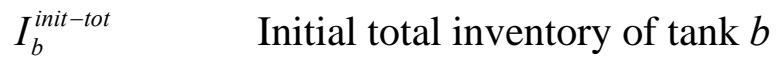

$I_{j b}^{i n i t} \quad$ Initial inventory of component $j$ in $\operatorname{tank} b$

$I_{b}^{L} \quad$ Lower bound on total inventory in a tank $b$

$I_{b}^{U} \quad$ Upper bound on total inventory in a tank $b$

ND Number of distillation units in the network

$\mathrm{ND}_{\mathrm{D} 1} \quad$ Number of distillation units in sub-structure D1

$\mathrm{ND}_{\mathrm{D} 2} \quad$ Number of distillation units in sub-structure D2

NE Number of transfer events

$T_{p}^{\text {arrival }} \quad$ Arrival time of crude in supply stream $p$

$V_{a b}^{L} \quad$ Lower bound on flow from $a$ to $b$

$V_{b c}^{L} \quad$ Lower bound on flow from $b$ to $c$

$V_{a b}^{U} \quad$ Upper bound on flow from $a$ to $b$

$V_{b c}^{U} \quad$ Upper bound on flow from $b$ to $c$

$V_{p}^{\text {supply }} \quad$ Total volume of crude oil arriving in supply stream $p$

$\lambda_{m n t}^{V t o t} \quad$ Lagrange multiplier

$\lambda_{\text {jmnt }}^{V C} \quad$ Lagrange multiplier

$\lambda_{m n t}^{T 1} \quad$ Lagrange multiplier

$\lambda_{m n t}^{T 2} \quad$ Lagrange multiplier

$\lambda_{m n t}^{w} \quad$ Lagrange multiplier 


\section{Continuous Variables}

$I_{b t}^{\text {tot }} \quad$ Total inventory of tank $b$ at the end of transfer event $t$

$I_{j b t} \quad$ Inventory of component $j$ in tank $b$ at the end of transfer event $t$

$T_{a b t}^{1} \quad$ Starting time of a transfer from $a$ to $b$ in transfer event $t$

$T_{b c t}^{1} \quad$ Starting time of a transfer from $b$ to $c$ in transfer event $t$

$T_{a b t}^{2} \quad$ Ending time of a transfer from $a$ to $b$ in transfer event $t$

$T_{b c t}^{2} \quad$ Ending time of a transfer from $b$ to $c$ in transfer event $t$

$T_{p}^{\text {start }} \quad$ Initial starting time of crude transfer from supply stream $p$

$T_{p}^{\text {end }} \quad$ Overall ending time of crude transfer from supply stream $p$

$V_{a b t}^{t o t} \quad$ Total flow from $a$ to $b$ in transfer event $t$

$V_{b c t}^{\text {tot }} \quad$ Total flow from $b$ to $c$ in transfer event $t$

$V_{\text {jabt }}^{\text {tot }} \quad$ Flow of component $j$ from $a$ from $b$ in transfer event $t$

$V_{j b c t}^{t o t} \quad$ Flow of component $j$ from $b$ from $c$ in transfer event $t$

\section{Binary variables}

$w_{a b t} \quad$ Equal to 1 if there is a flow from $a$ to $b$ in transfer event $t$ else 0

$w_{b c t} \quad$ Equal to 1 if there is a flow from $b$ to $c$ in transfer event $t$ else 0

\section{References}

1. Adjiman, C. S.; Androulakis, I. P.; Floudas, C. A. (2000): Global Optimization of Mixed-Integer Nonlinear Problems. American Institute of Chemical Engineering Journal, 46, 1769 -1797.

2. Bergamini, M. L.; Aguirre, P.; Grossmann, I. E. (2005). Logic-based outer approximation for globally optimal synthesis of process networks. Computers and Chemical Engineering, 29, 1914 1933.

3. Brooke, A.; Kendrick, D.; Meeraus, A; Raman, R. (1998). GAMS: A User’s Guide, Release 2.50. GAMS Development Corporation.

4. Duran, M. A.; Grossmann, I. E. (1986). An Outer-Approximation Algorithm for a Class of Mixed-Integer Nonlinear Programs. Mathematical Programming, 36, 307 -339. 
5. Fisher, M. L. (1981). The Lagrangian Relaxation Method for solving Integer Programming Problems. Management Science, 27, 1 -18

6. Fletcher, R.; Leyffer, S. (1994). Solving Mixed Integer Nonlinear Programs by Outer Approximation. Mathematical Programming, 66, 327 -349.

7. Floudas, C. A.; Lin, X. (2004). Continuous-Time versus Discrete-Time Approaches for Scheduling of Chemical Processes: A Review. Computers and Chemical Engineering, 28, 2109 2129.

8. Furman, K. C.; Jia, Z.; Ierapetritou, M. G. (2006). A Robust Event-Based Continuous Time Formulation for Tank Transfer Scheduling. Work in Progress.

9. Grossmann, I. E. (2002). Review of Nonlinear Mixed-Integer and Disjunctive Programming Techniques. Optimization and Engineering, 3, 227 -252.

10. Jackson, J. R.; Grossmann, I. E. (2003). Temporal Decomposition Scheme for Nonlinear Multisite Production Planning and Distribution Models. Industrial and Engineering Chemistry Research, 42, $3045-3055$.

11. Jia, Z.; Ierapetritou, M. G.; Kelly, J. D. (2003). Refinery Short-Term Scheduling Using Continuous Time Formulation: Crude Oil Operations. Industrial and Engineering Chemistry Research, 42, 3085 -3097.

12. Karuppiah, R.; Grossmann, I. E. (2006). A Lagrangean based Branch-and-Cut algorithm for global optimization of nonconvex Mixed-Integer Nonlinear Programs with decomposable structures, Submitted to Journal of Global Optimization.

13. Kesavan, P.; Allgor, R. J.; Gatzke, E. P.; Barton, P. I. (2004). Outer Approximation Algorithms for Separable Nonconvex Mixed-Integer Nonlinear Programs. Mathematical Programming, 100, $517-535$.

14. Lee, H.; Pinto, J. M.; Grossmann, I. E.; Park, S. (1996). Mixed Integer Linear Programming Model for Refinery Short-Term Scheduling of Crude Oil Unloading with Inventory Management. Industrial and Engineering Chemistry Research, 35, 1630 -1641.

15. McCormick, G. P. (1976). Computability of Global Solutions to Factorable Nonconvex Programs - Part I - Convex Underestimating Problems. Mathematical Programming, 10, 146 -175.

16. Mendez, C. A.; Cerda, J.; Grossmann, I. E.; Harjunkoski, I; Fahl, M. (2006). State-of-the-art review of optimization methods for short-term scheduling of batch processes. Computers and Chemical Engineering, 30, 913 -946.

17. Pinto, J. M.; Joly, M.; Moro, L. F. L. (2000). Planning and Scheduling Models for Refinery Operations. Computers and Chemical Engineering, 24, 2259 -2276.

18. Sahinidis, N. (1996). BARON: A General Purpose Global Optimization Software Package. Journal of Global Optimization, 8, 201 -205. 
19. Shah, N. (1996). Mathematical Programming Techniques for Crude Oil Scheduling. Computers and Chemical Engineering (Suppl.), 20, S1227 -S1232.

20. Sherali, H. D.; Alameddine, A. (1992). A New Reformulation Linearization Technique for Bilinear Programming Problems. Journal of Global Optimization, 2, 379-410.

21. Tawarmalani, M.; Sahinidis, N. (2002). Convexification and Global Optimization in Continuous and Mixed-Integer Nonlinear Programming: Theory, Algorithms, Software and Applications. Kluwer Academic Publishers : Dordrecht, The Netherlands

22. Tawarmalani, M.; Sahindis, N. V. (2004). Global Optimization of Mixed-Integer Nonlinear Programs: A Theoretical and Computational Study. Mathematical Programming, 99, 563 -591.

23. Wei, J.; Furman, K. C.; Duran, M. A.; Tawarmalani, M.; Sahinidis, N. V. (2005) Global Optimization for Stochastic Nonconvex Mixed Integer Nonlinear Programs. IFORS 2005, Honolulu, Hawaii.

24. Zamora, J. M.; Grossmann, I. E. (1999). A Branch and Bound Algorithm for Problems with Concave Univariate, Bilinear and Linear Fractional Terms. Journal of Global Optimization, 14 (3), $217-249$.

\section{Appendix : Updating Lagrange multipliers}

Fisher (1981) proposed a sub-gradient method to update Lagrange multipliers, to be used in solving a Lagrangean relaxation of the original problem, starting from an initial arbitrary value of the multipliers. This technique is tailored to suit our problem in order to obtain updated values of Lagrange multipliers starting with random initial values. The generated Lagrange multipliers are then used to derive cuts to be added to the relaxation. For solving the models (LD1-R) and (LD2-R) resulting from a decomposition of the network structure into sub-structures D1 and D2, we succesively generate the multipliers as follows:

$$
\begin{array}{ll}
{\left[\lambda_{m n t}^{\text {Vtot }}\right]^{k+1}=\left[\lambda_{m n t}^{\text {Vtot }}\right]^{k}+t s^{k}\left[\left(V_{m n t}^{t o t, 1}\right)^{* k}-\left(V_{m n t}^{t o t, 2}\right)^{* k}\right]} & \forall m \in M, \forall n \in N_{m}, \forall t \in T \\
{\left[\lambda_{j m n t}^{V C}\right]^{k+1}=\left[\lambda_{j m n t}^{V C}\right]^{k}+t s^{k}\left[\left(V_{j m n t}^{1}\right)^{* k}-\left(V_{j m n t}^{2}\right)^{* k}\right]} & \forall j \in J, \forall m \in M, \forall n \in N_{m}, \forall t \in T \\
{\left[\lambda_{m n t}^{T 1}\right]^{k+1}=\left[\lambda_{m n t}^{T 1}\right]^{k}+t s^{k}\left[\left(T_{m n t}^{1,1}\right)^{* k}-\left(T_{m n t}^{1,2}\right)^{* k}\right]} & \forall m \in M, \forall n \in N_{m}, \forall t \in T \\
{\left[\lambda_{m n t}^{T 2}\right]^{k+1}=\left[\lambda_{m n t}^{T 2}\right]^{k}+t s^{k}\left[\left(T_{m n t}^{2,1}\right)^{* k}-\left(T_{m n t}^{2,2}\right)^{* k}\right]} & \forall m \in M, \forall n \in N_{m}, \forall t \in T
\end{array}
$$


$\left[\lambda_{m n t}^{w}\right]^{k+1}=\left[\lambda_{m n t}^{w}\right]^{k}+t s^{k}\left[\left(w_{m n t}^{1}\right)^{* k}-\left(w_{m n t}^{2}\right)^{* k}\right] \quad \forall m \in M, \forall n \in N_{m}, \forall t \in T$

where $t s^{k}$ is a scalar step size and $\left\{\left(V_{m n t}^{t o t, 1}\right)^{* k},\left(V_{j m n t}^{1}\right)^{* k} \forall j,\left(T_{m n t}^{1,1}\right)^{* k},\left(T_{m n t}^{2,1}\right)^{* k},\left(w_{m n t}^{1}\right)^{* k}\right\}$ and $\left\{\left(V_{m n t}^{t o t, 2}\right)^{* k},\left(V_{j m n t}^{2}\right)^{* k} \forall j,\left(T_{m n t}^{1,2}\right)^{* k},\left(T_{m n t}^{2,2}\right)^{* k},\left(w_{m n t}^{2}\right)^{* k}\right\}$ are the optimal values of the duplicate variables, at the kth iteration, obtained from the solution of the sub-problems (LD1-R) and (LD2-R), respectively. The following formula is used to calculate the values of $t s^{k}$ at every iteration $k$ :

$$
t s^{k}=\frac{\alpha^{k}\left(z^{U}-z^{L}\left(\lambda^{k}\right)\right)}{\sum_{m \in M} \sum_{n \in N_{m}} \sum_{t}\left[\left(\left(V_{m n t}^{t o t, 1}\right)^{* k}-\left(V_{m n t}^{t o t, 2}\right)^{* k}\right)+\sum_{j}\left(\left(V_{j m n t}^{1}\right)^{* k}-\left(V_{j m n t}^{2}\right)^{* k}\right)+\left(\left(T_{m n t}^{1,1}\right)^{* k}-\left(T_{m n t}^{1,2}\right)^{* k}\right)+\left(\left(T_{m n t}^{2,1}\right)^{* k}-\left(T_{m n t}^{2,2}\right)^{* k}\right)+\left(\left(w_{m n t}^{1}\right)^{* k}-\left(w_{m n t}^{2}\right)^{* k}\right)\right]}
$$

where $\alpha^{k}$ is a scalar chosen between 0 and 2, $z^{L}\left(\lambda^{k}\right)$ is the sum of the obejctives of the sub-models (LD1-R) and (LD2-R), when the multipliers are set to $\lambda^{k}$, and $z^{U}$ is the value of the best found feasible solution to $(\mathrm{R})$. The parameter values used for updating the multipliers in the numerical examples in the paper is given in Table 5c. The multiplier update was carried out only once in this work. The updating scheme remains the same if the network is decomposed into sub-structures D3 and D4 and the sub-models pertaining to those are to be solved. 